\title{
Dynamic Model and Dynamic Response of Automobile Dual-Mass Flywheel with Bifilar-Type Centrifugal Pendulum Vibration Absorber
}

\author{
Lei Chen $\mathbb{D}^{1},{ }^{1}$ Jianming Yuan $\mathbb{D}^{2},{ }^{2}$ Hang Cai, ${ }^{3}$ and Jinmin $\mathrm{Hu}^{4}$ \\ ${ }^{1}$ School of Mechanical and Electronic Engineering, Wuhan University of Technology, Wuhan 430070, China \\ ${ }^{2}$ School of Logistics Engineering, Wuhan University of Technology, Wuhan 430070, China \\ ${ }^{3}$ HaiTong Investment Group, Chongqing 404100, China \\ ${ }^{4}$ Shenzhen Road Rover Technology Co., Ltd, Shenzhen 518000, China \\ Correspondence should be addressed to Jianming Yuan; whtu_yjm@163.com
}

Received 15 October 2020; Revised 20 January 2021; Accepted 15 February 2021; Published 17 May 2021

Academic Editor: Chengzhi Yuan

Copyright (c) 2021 Lei Chen et al. This is an open access article distributed under the Creative Commons Attribution License, which permits unrestricted use, distribution, and reproduction in any medium, provided the original work is properly cited.

Compared with dual-mass flywheel (DMF) and DMF with simple-type centrifugal pendulum vibration absorber (CPVA), DMF with bifilar-type CPVA has a better damping performance in the whole speed range of engine. The related research mainly focused on local models, such as dynamic model of DMF and dynamic model of CPVA, and the effect of the curvature path of CPVA on the damping performance. The reported models and methods are not sufficient for the system of DMF coupled with bifilar CPVA. Aiming at the deficiency of local models and the limitation of bench test, an integral model for DMF with bifilar CPVA is proposed and the real vehicle test is carried out in this study. Involving the moment of inertia of the centrifugal pendulum, the model considers the nonlinearities of DMF and bifilar CPVA. Afterward, the dynamic model of the automobile power transmission system equipped with the DMF with bifilar-type CPVA is built, and the dynamic responses of the system are investigated under idling and driving conditions. According to the simulation results, DMF with bifilar-type CPVA shows better vibration reduction performance in full-speed range. Moreover, the key structural parameters $R$ and $l$ influencing the damping performance of DMF with bifilar CPVA are discussed. The results show that the sum of $R$ and $l$ is directly proportional to the damping effect. Finally, real vehicle tests under idling and driving conditions (engine speed from $750 \mathrm{r} / \mathrm{min}$ to $3400 \mathrm{r} / \mathrm{min}$ ) are carried out. The test results show that the $2^{\text {nd }}$ order engine speed fluctuations are attenuated by more than $80 \%$ by DMF with bifilar CPVA with engine speed lower than $2000 \mathrm{r} / \mathrm{min}$ and are attenuated by more than $90 \%$ with engine speed higher than $2000 \mathrm{r} / \mathrm{min}$. The experimental results are basically consistent with the simulation results, which verify the validity of the model.

\section{Introduction}

There are many sources of vehicle vibrations and noises, such as the engine, the tire, the transmission, and the road surface [1], in which the engine contributes the most [2]. In addition, the vibrations and noises caused by the engine will further produce transmission vibrations and noises [3]. Global emission regulation requires the automotive manufacturers to develop engines with lower level emissions. The development of the turbocharged three-cylinder engine is a strategy to meet this goal. However, the natural structural characteristics bring a greater challenge on $\mathrm{NVH}$ performance than the four-cylinder engine [4]. In order to attenuate the torsional vibrations caused by the engine, torsional vibration dampers are employed to vehicle power transmissions.

Palliative devices, such as clutch predampers and dualmass flywheel, have been used to mitigate the effect of transmitted engine torsional oscillations [5]. Equipping a clutch predamper (CTD) is the traditional way to attenuate the torsional vibration of the powertrain. However, limited by the space and the large stiffness of the elastic element, the damping effect is poor [6]. As a new kind of automobile torsional damper, DMF (dual-mass flywheel) has the 
functions of the single-mass flywheel and the CTD [7]. With reasonable mass distribution and torsional stiffness, a DMF can reduce the natural frequency of the powertrain below the common speed and thus attenuate the torsional vibrations under idling and driving conditions [8]. The circumferential long arc spring-type DMF is the most widely used, and the technology is the most mature [9]. The structural characteristics and working principle of DMF determine that the $\mathrm{DMF}$ is suitable for low-speed vibration reduction, but the damping performance of the DMF in the high-speed region is decreased [10].

The natural frequency of the centrifugal pendulum damper (CPVA) is related to the rotational speed, which shows excellent damping performance in the whole speed range and has been widely used in the aviation field [11]. The centrifugal pendulums have been applied to the large torsional angle clutch and DMF since 2008, which can attenuate the vibration of the main harmonic excitation of the engine. Cirelli [11] analyzed the variation law of velocity and acceleration of a parallel and trapezoidal bifilar centrifugal pendulum from the perspective of kinematics. Accordingly, a linear dynamic model of bifilar centrifugal pendulum was developed. Some studies by Li Wei and Long Yan [12] suggested that the natural frequency of a DMF with CPVA was proportional to the rotating speed, and the vibration of engine fire frequencies could be absolutely eliminated theoretically by adjusting the parameters of the centrifugal pendulum. Wu Huwei and Wu Guangqiang [13] found that the large angular clutch with CPVA could reduce not only the natural frequency of the vehicle powertrain but also the torsional vibration amplitude of the engine. Hässler and Kooy [14] experimentally investigated the damping performance of a DMF and a clutch with CPVA. They discovered that the clutch with CPVA showed a better damping performance than the DMF in the speed region above $2000 \mathrm{r} / \mathrm{min}$, whereas the result was reversed when the engine speed was below $2000 \mathrm{r} / \mathrm{min}$. Seong-Young Song [15] established a linear dynamic model of a clutch with simple CPVA. The model was employed to analyze the dynamic response, and the results showed that the clutch with CPVA could attenuate the torsional vibration of vehicle powertrain. The experimental results also demonstrated the finding. Chen Long and Shi Wenku [16] created the simulation models of DMF, clutch, and DMF with CPVA, and they found the DMF with CPVA possessed the best damping performance. Rao and Sujatha [17] proposed the design strategy to reduce the $1^{\text {st }}$ and $2^{\text {nd }}$ order axial vibrations by using circular path pendulum absorbers and analytically solved the equations of motion at the $2^{\text {nd }}$ order. The authors in [18] analyzed the stability of a simple CPVA and a bifilar CPVA from the perspective of kinematics. They found that the simple CPVA was prone to instability due to the large swing angle in the high-speed range, while the bifilar CPVA showed a better angle constraint and better stability. Shi and Parker [19] developed an analytical model of CPVA systems with equally spaced, identical absorbers and investigated the structure of the modal vibration properties, and then, the critical speeds and flutter instability of the system were studied numerically and analytically based on the model.
Marco Cirelli et al. [20] applied the methodology of Desoyer and Slibar to solve the dynamics of the centrifugal pendulum with cycloidal and epicycloidal pendulum paths, and the numerical simulations confirmed the better damping capabilities of the cycloidal and epicycloidal centrifugal pendula with respect to the classic circular path. Mayet and Ulbrich [21] proposed a general approach for the design of tautochronic pendulum vibration absorbers, and the method could deal with a large variety of nonbifilar centrifugal vibration absorber designs, which provide application-related optimal performance and resolve some of the existing design limitations. The authors in [22] provided an analytical proof of the optimal tuning of centrifugal pendulum vibration absorbers (CPVAs) to reduce in-plane translational and rotational vibration for a rotor with $N$ cyclically symmetric substructures attached to it, and the solutions showed that the rotor translational vibration at order $j$ was reduced when one group of CPVAs was tuned to order $j_{N-1}$ and the other was tuned to order $j_{N+1}$. Pier Paolo Valentin and Marco Cirelli et al. [23] analyzed a methodology for designing compliant centrifugal dampers based on the arrangement of a collection of leaf flexure hinges connecting peripheral masses. The pseudorigid surrogate model was deduced taking into account second-order kinematic invariants and Euler-Savary equations, thus providing second-order approximation of the relative motion.

The above literatures show that the natural frequency of the centrifugal pendulum torsional damper is correlated with the engine speed. In addition, the harmonic order of the torsional vibration can be tuned when the ratio of $l$ to $R$ is equal to a certain harmonic order of the engine, in which $l$ is the distance of the center of mass to the suspension point of the CPVA and $R$ represents the distance of the connecting point of the CPVA to the rotating center. It is clear that a number of the combinations of $l$ and $R$ can meet the above tuning requirement. Therefore, whether the different combinations of $l$ and $R$ affect the damping performance needs to be discussed. Both DMF and CPVA show nonlinear dynamic characteristics. When they are combined together, the dynamic model of the whole system should consider their dynamic characteristics. Since the damping performance should be observed in the powertrain system, the dynamic model of the powertrain system equipping the DMF with CPVA should be developed. Recently, the CPVA and DMF are usually studied separately. Furthermore, most of the studies focus on local linear models of the CPVA, and the studies focusing on the dynamic responses of the powertrain involving CPVA are rarely mentioned. During the operation of the CPVA, some literatures [12] suggested that the moment of inertia could be neglected when modeling CPVA since the mass was so tiny. Nevertheless, the mass of the bifilar CPVA can theoretically be designed to be larger than that of the simple CPVA [18]; hence, the moment of inertia of the bifilar CPVA cannot be neglected. In the aspect of model validation, most of the research studies only give numerical simulation, bench test for local model of a shock absorber, and low-speed vehicle test.

According to the research results of the above literatures, there are two main problems that need to be supplemented, 


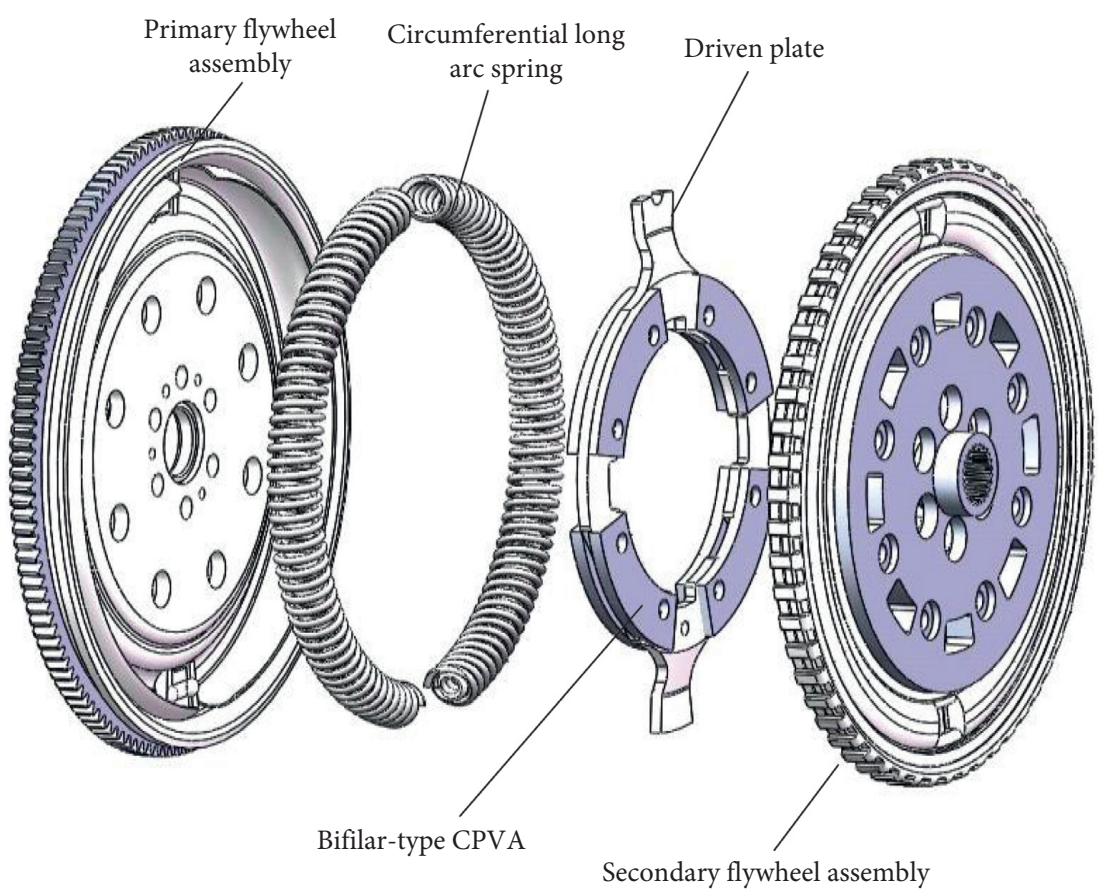

Figure 1: Schematic diagram of the DMF with bifilar CPVA.

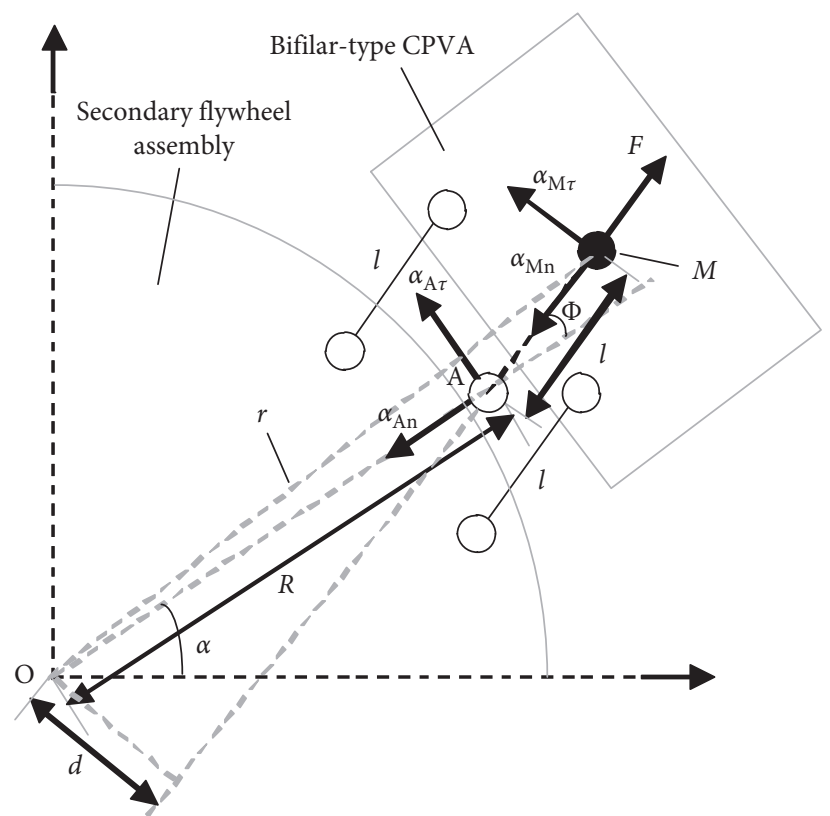

FIgURE 2: Simplified model of the bifilar CPVA. O: rotary center of the secondary flywheel; $A$ : equivalent connection point of the CPVA; $M$ : center of mass of CPVA; $\alpha$ : rotation angle of the secondary flywheel; $\phi$ : the swing angle of the CPVA relative to the secondary flywheel; $R$ : distance between the equivalent connecting point of the CPVA and the rotary center of secondary flywheel; $l$ : distance from the equivalent connecting point of the CPVA to its centroid; $F$ : centrifugal force of the CPVA; $d$ : force arm of centrifugal force; $r$ : distance from the centroid of the CPVA to point; $m$ : mass of the bifilar CPVA.

that is, the dynamic model of DMF with bifilar CPVA and the model validation. As for the dynamic model of DMF with bifilar CPVA, there are few published literatures about DMF and CPVA as an ensemble; in fact, DMF and CPVA need to be analyzed as an entirety, which means the integral dynamic model should contain the dynamic models of DMF and bifilar CPVA. Moreover, the different combinations of $l$ and $R$ affecting the damping performance must be discussed. With regard to model validation, numerical simulation, bench test for local model of a shock absorber, and lowspeed vehicle test were applied to verify the dynamic model in the above literatures; theoretically, it is more sufficient 
that the real vehicle experiment covering full working speed of engine as far as possible is used to verify the effectiveness of the model.

The objective of this study is to establish the integral dynamic model of the powertrain matching DMF with bifilar CPVA and consummate the model validation. Firstly, a nonlinear integral dynamic model involving the moment of inertia of the CPVA, the nonlinearity of bifilar CPVA, and the nonlinearity of DMF is developed. Then, the model is used in modeling the automobile power transmission system. Additionally, the damping performance of the DMF with bifilar CPVA is theoretically investigated, and the influence of different combinations of $R$ and $l$ on the damping performance is discussed. Finally, the model is validated by real vehicle tests covering the full working speed range of engine (from $750 \mathrm{r} / \mathrm{min}$ to $3400 \mathrm{r} / \mathrm{min}$ ).

\section{Linear Dynamic Model of Secondary Flywheel with a Bifilar CPVA}

A basic DMF consists of two separated flywheel assemblies connected by a spring-damping damper, as shown in Figure 1 . The primary flywheel assembly mainly includes a starting gear ring, a signal ring, a cover, and a primary flywheel. The secondary flywheel assembly mainly comprises a driven plate, a seal disc, and a secondary flywheel. The primary assembly is connected to the engine crankshaft, and the secondary assembly is connected to the clutch. Thus, power from the engine can be initially transmitted to the primary assembly and then to the secondary assembly by compressing the arc springs through the driven plate. Finally, the power reaches the power transmission leading to car driving. As shown in Figure 1, on the basis of the structure of the DMF, the bilifar CPVAs are symmetrically installed on the driven plate in circumferential direction.

With reference to Figure 2, the dynamic equations for the bifilar-type CPVA have been deduced as follows:

$$
\begin{aligned}
a_{A \tau} & =R \ddot{a}, \\
a_{A n} & =R \dot{a}^{2}, \\
a_{M \tau} & =l(\ddot{a}+\ddot{\phi}), \\
a_{M n} & =l(\dot{a}+\dot{\phi})^{2},
\end{aligned}
$$

where $a_{A \tau}$ and $a_{M \tau}$ denote the tangential acceleration of $A$ and $M$ relative to $A$, respectively, and $a_{A n}$ and $a_{M n}$ represent the normal acceleration of $A$ and $M$ relative to $A$, respectively.

The absolute tangential acceleration of $\mathrm{M} \cdot a_{M \tau}^{0}$ can be expressed as follows $[18,19]$ :

$$
a_{M \tau}^{0}=a_{M \tau}+a_{A \tau} \cos (\phi)+a_{A n} \sin (\phi) .
$$

Combining equations (1), (2), (3), and (4) with equation (5), $a_{M \tau}^{0}$ can be rewritten as

$$
a_{M \tau}^{0}=l(\ddot{a}+\ddot{\phi})+R \ddot{a} \cos (\phi)+R \dot{a}^{2} \sin (\phi) \text {. }
$$

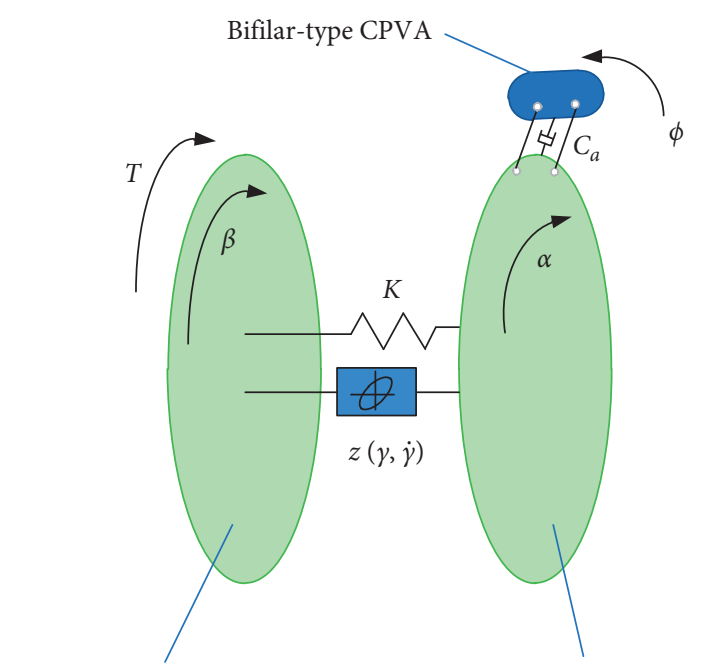

Primary flywheel assembly

Secondary flywheel assembly

Figure 3: Dynamic model of the DMF with a bifilar-type CPVA.

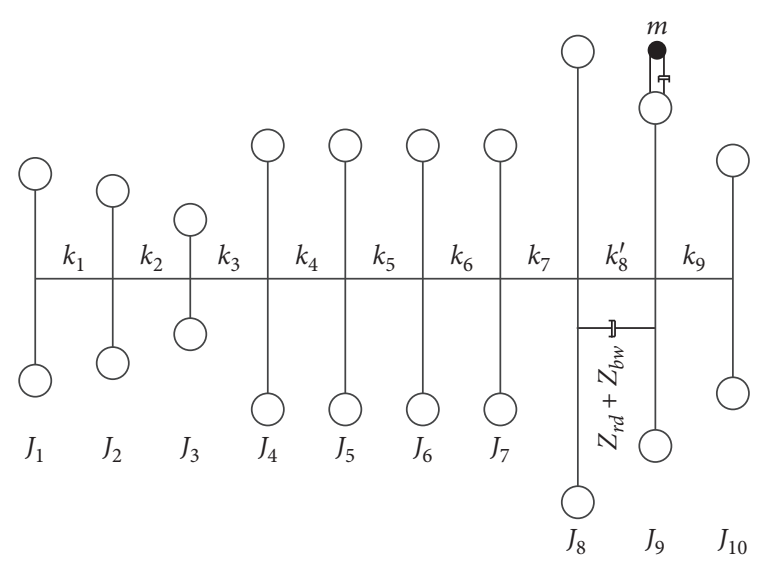

FIGURE 4: Torsional vibration model of power transmission under the idling condition.

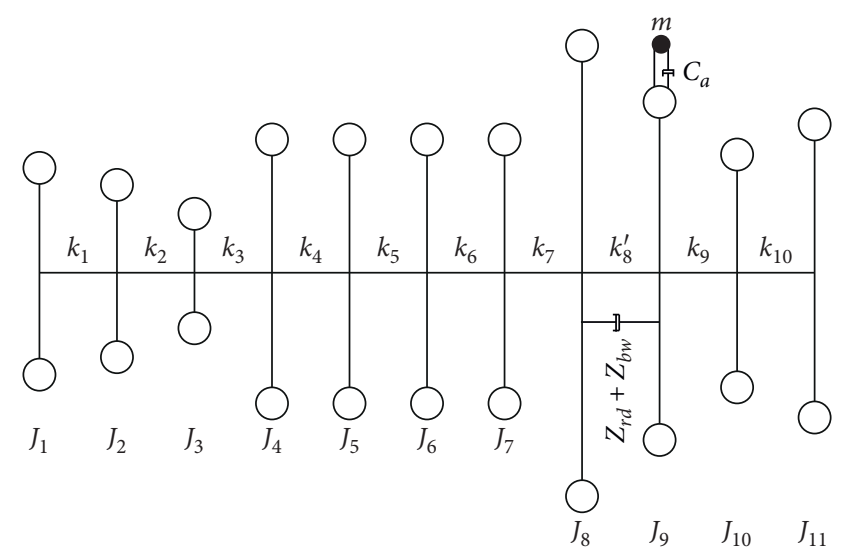

FIgURE 5: Torsional vibration model of power transmission under the driving condition.

Thus, the equation of motion of the bilifar CPVA can be given by 
TABLE 1: Structural parameters of the power transmission.

\begin{tabular}{lcccc}
\hline Names of elements & Inertia & $\begin{array}{c}\text { Value of inertia } \\
\left(\mathrm{Kg} \bullet \mathrm{m}^{2}\right)\end{array}$ & $\begin{array}{c}\text { Torsional } \\
\text { stiffness }\end{array}$ & Value of torsional stiffness $(\mathrm{N} \bullet \mathrm{m} / \mathrm{rad})$ \\
\hline $\begin{array}{l}\text { Driven part of rubber } \\
\text { damper }\end{array}$ & $J_{1}$ & $4.795 E-3$ & $K_{1}$ & 14320 \\
$\begin{array}{l}\text { Driving part of rubber } \\
\text { damper }\end{array}$ & $J_{2}$ & $2.038 E-3$ & $K_{2}$ & 74636 \\
Accessories & $J_{3}$ & $9.74 E-5$ & $K_{3}$ & 356181 \\
Cylinder 1 & $J_{4}$ & $4.715 E-3$ & $K_{4}$ & 358936 \\
Cylinder 2 & $J_{5}$ & $4.712 E-3$ & $K_{5}$ & 361005 \\
Cylinder 3 & $J_{6}$ & $4.712 E-3$ & $K_{6}$ & 359750 \\
Cylinder 4 & $J_{7}$ & $4.69 E-3$ & $K_{7}$ & 1872000 \\
Primary flywheel assembly & $J_{8}$ & 0.075 & $K_{8}^{\prime}$ & 165 (under idling condition); 750 (under driving \\
Secondary flywheel assembly & $J_{9}$ & 0.0125 & $K_{9}$ & 99213 \\
Input shaft of gearbox & $J_{10}$ & $7.312 E-3$ & $K_{10}$ & 48650 \\
Transmission shaft system & $J_{11}$ & 0.02684 & & \\
\hline
\end{tabular}

TABLE 2: Model parameters of the DMF with the bifilar CPVA.

\begin{tabular}{lccc}
\hline Names of elements & Value & Unit & Remark \\
\hline$R$ & 76 & $\mathrm{~mm}$ & $\mathrm{~mm}$ \\
$l$ & 19 & $\mathrm{Kg}$ & $\mathrm{Kg} \cdot \mathrm{m}^{2}$ \\
$m$ & 1 & $\mathrm{~N} \cdot \mathrm{m} /(\mathrm{rad} / \mathrm{s})$ & $\mathrm{N} \cdot \mathrm{m} /(\mathrm{rad} / \mathrm{s})$ \\
$I$ & 0.004 & $\mathrm{Null}$ & Null \\
$C_{a}$ & 0.1 & Null & Engine speed $<1000 \mathrm{r} / \mathrm{min}$ \\
$C_{s}$ & 0.1 & Null & Null \\
$\mu$ & -0.1022 & Null & Engine speed $\geq 2000 \mathrm{r} / \mathrm{min}$ \\
$\lambda$ & 0.59185 & Null & \\
$\eta$
\end{tabular}

TABLE 3: Simulation algorithm.

Algorithm

Step 1: identify parameters of the Bouc-Wen model $(\mu, \lambda, \eta, \mathbf{b}, \mathbf{c})$ from equation (27) based on test data and then obtain $\mathbf{Z}(\gamma, \dot{\gamma})$

Step 2: prepare parameters in Tables 1 and 2, engine speed $\omega_{e}$, and excitation torque $T_{1}, T_{2}, T_{3}, T_{4}$

Step 3: construct the vector Y of state variables of the system $Y=\left[\begin{array}{c}\alpha_{1} \\ \dot{\alpha}_{1} \\ \vdots \\ \phi \\ \dot{\phi} \\ \vdots \\ \alpha_{11} \\ \dot{\alpha}_{11}\end{array}\right]$

Step 4: establish the system state equation from equations (45) and (46)

Step 5: use Runge-Kutta algorithm to solve the system state equation in Matlab software platform

$$
m\left(l(\ddot{a}+\ddot{\phi})+R \ddot{a} \cos (\phi)+R \dot{a}^{2} \sin (\phi)\right)=m g \sin (\phi) .
$$

Because the secondary flywheel rotary speed is approximately equal to the engine rotary speed, that is, $R \alpha^{2} \gg g$, then equation (7) can be reduced as

$$
\ddot{l} \ddot{\phi}+(l+R \cos (\phi)) \ddot{a}+R \dot{a}^{2} \sin (\phi)=0 .
$$

Assume that the average rotary speed of the secondary flywheel is $\mu$. Suppose that the amplitude and frequency of the rotary speed fluctuation of the secondary flywheel are $A_{0}$ and $\omega$, respectively. The rotation angle of the secondary flywheel is expressed as

$$
a=\mu t+A_{0} \sin (\omega t) .
$$

Assuming that $\mathrm{A}_{0}$ and $\Phi$ are tiny, then $\dot{\alpha} \approx \mu, \sin (\phi) \approx \phi$ and $\cos (\phi) \approx 1$; equation (8) can be obtained as

$$
\ddot{\phi}+\frac{R}{1} \mu^{2} \phi \frac{(1+R)}{1} A_{0} \omega^{2} \sin (\omega t) \text {. }
$$




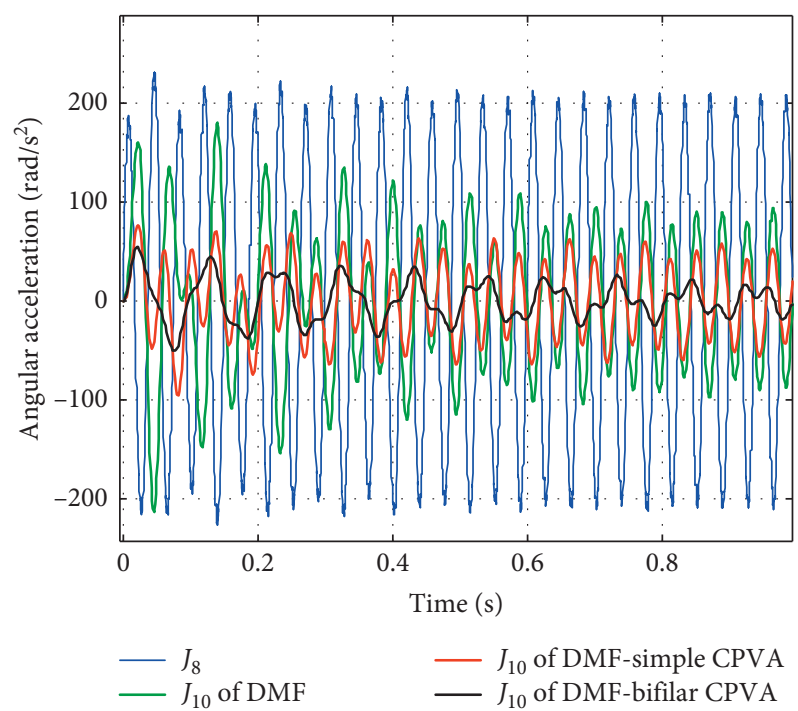

Figure 6: Angular acceleration curves of $J_{8}$ and $J_{10}$ under the idling condition.

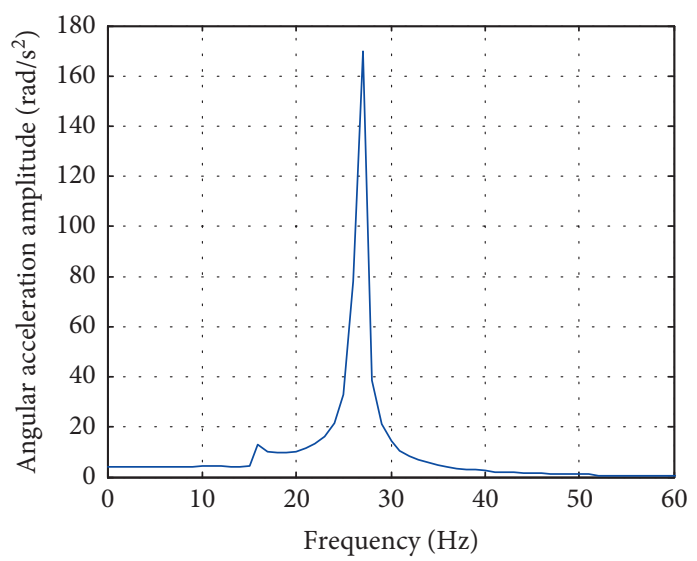

(a)

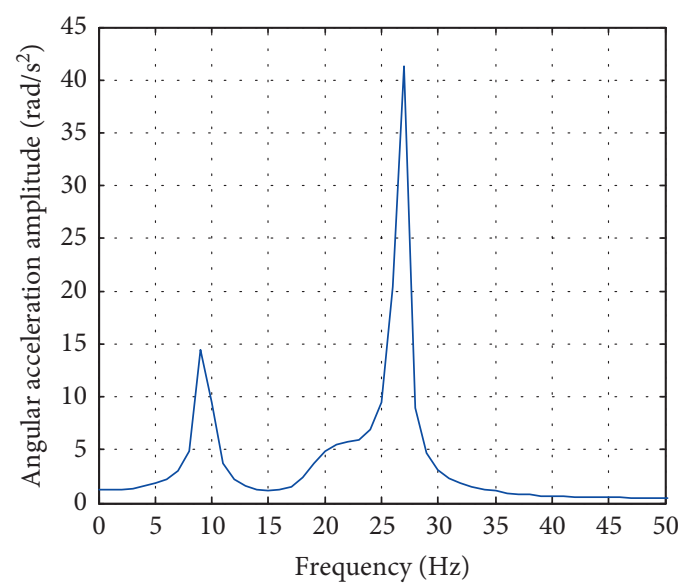

(c)

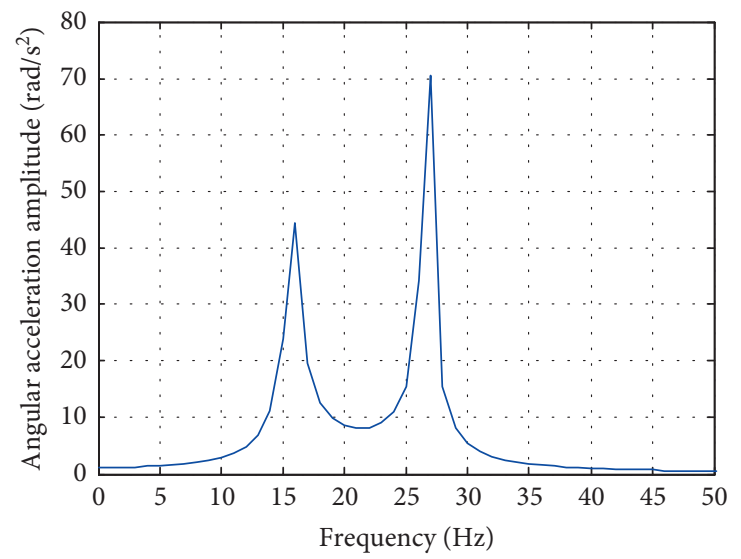

(b)

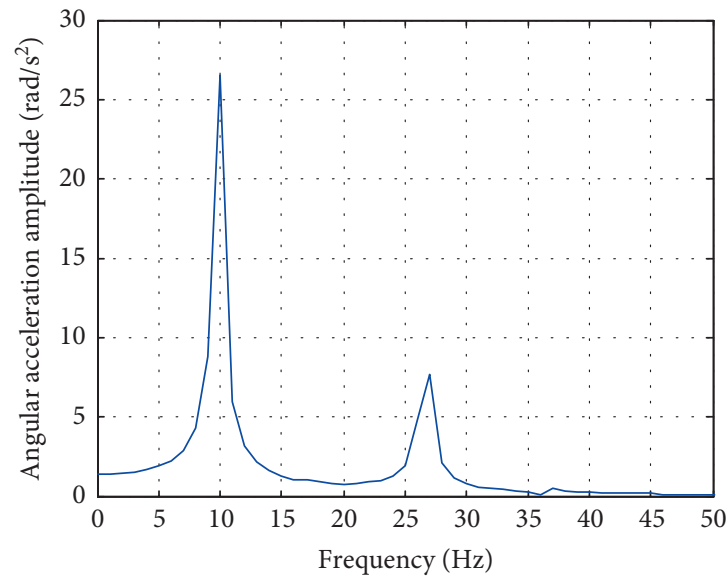

(d)

FIGURE 7: Spectra of $J_{8}$ and $J_{10}$ under the idling condition. (a) spectrum of $J_{8}$ and (b) spectrum of $J_{10}$ of DMF; (c) spectrum of $J_{10}$ of DMF with simple CPVA; (d) spectrum of $J_{10}$ of DMF with bifilar CPVA. 


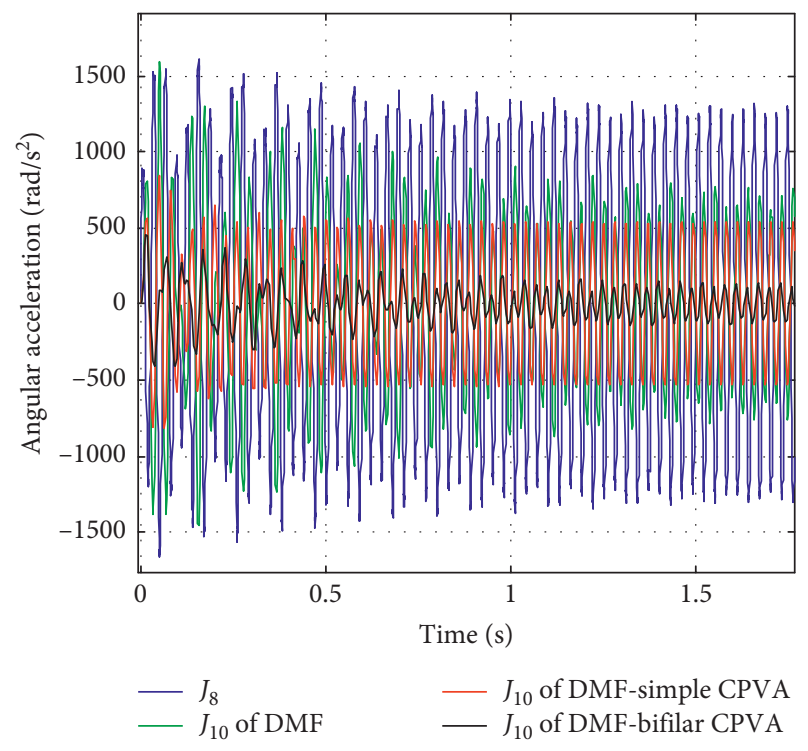

Figure 8: Angular acceleration curves of $J_{8}$ and $J_{10}$ at $1000 \mathrm{r} / \mathrm{min}$ of engine speed.

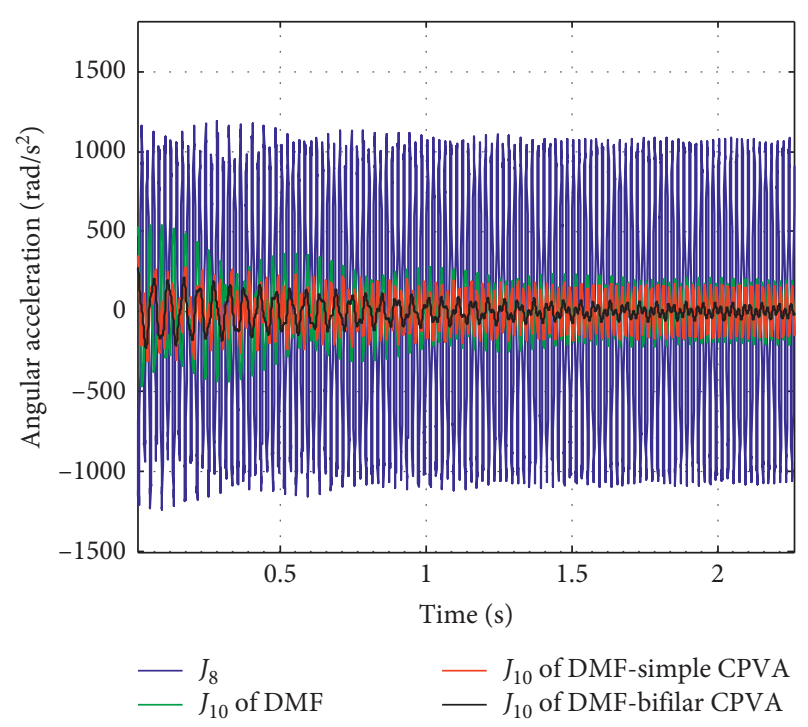

FIGURE 9: Angular acceleration curves of $J_{8}$ and $J_{10}$ at $1500 \mathrm{r} / \mathrm{min}$ of engine speed.

The steady-state solution of equation (10) is expressed as

$$
\ddot{\phi}=\frac{R+l}{R \mu^{2}-l \omega^{2}} A_{0} \omega^{2} \sin (\omega t)=-\frac{R+l}{R \mu^{2}-l \omega^{2}} \ddot{a} .
$$

Also, the natural frequency of the bifilar CPVA $\omega_{n}$ can be given by

$$
\omega_{n}=\mu \sqrt{\frac{R}{l}}
$$

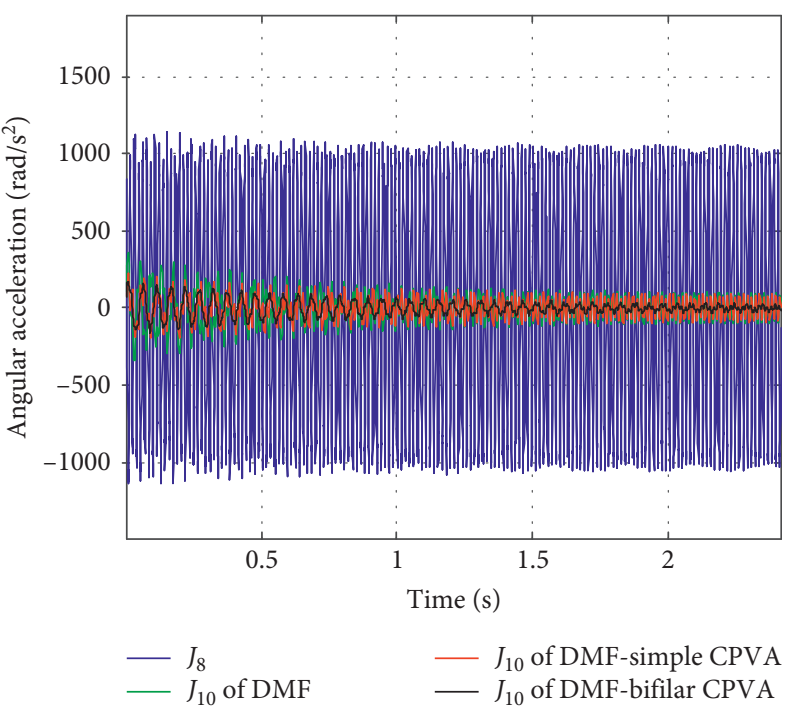

Figure 10: Angular acceleration curves of $J_{8}$ and $J_{10}$ at $2000 \mathrm{r} / \mathrm{min}$ of engine speed.

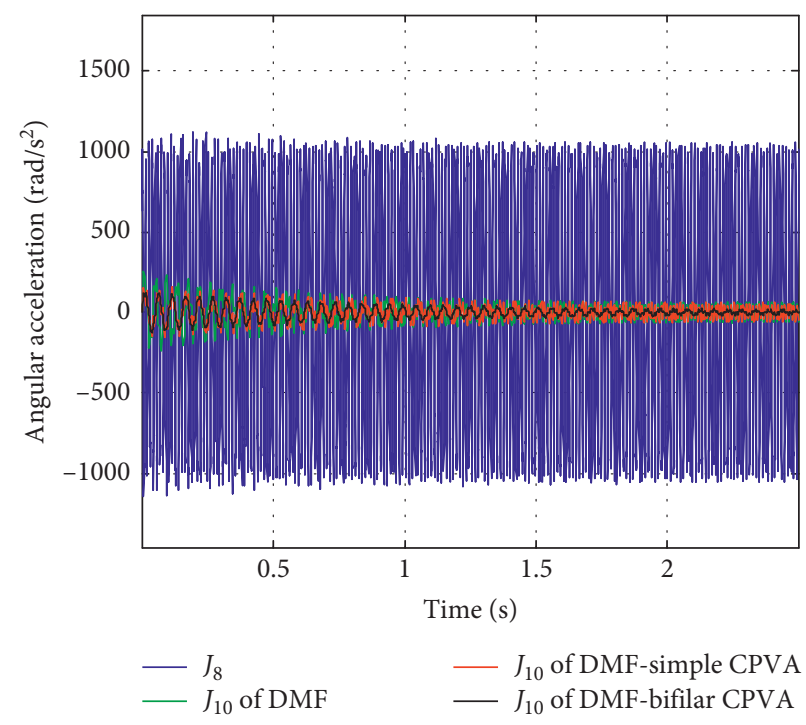

Figure 11: Angular acceleration curves of $J_{8}$ and $J_{10}$ at $2500 \mathrm{r} / \mathrm{min}$ of engine speed.

Referring to Figure 2, the centrifugal torque Tacting on the secondary flywheel by the bifilar CPVA can be described as

$$
T=m(\dot{a}+\dot{\phi})^{2} r d
$$

where

$$
\begin{aligned}
& r=\sqrt{R^{2}+l^{2}-2 R l \cos (\pi-\phi)} . \\
& d=R \sin (\phi) .
\end{aligned}
$$

Let $\sin (\phi) \approx \phi, \cos (\phi) \approx 1, \quad \dot{\alpha} \approx \mu, \mu \gg \dot{\phi}, \dot{\alpha} \gg \dot{\phi}$, then 


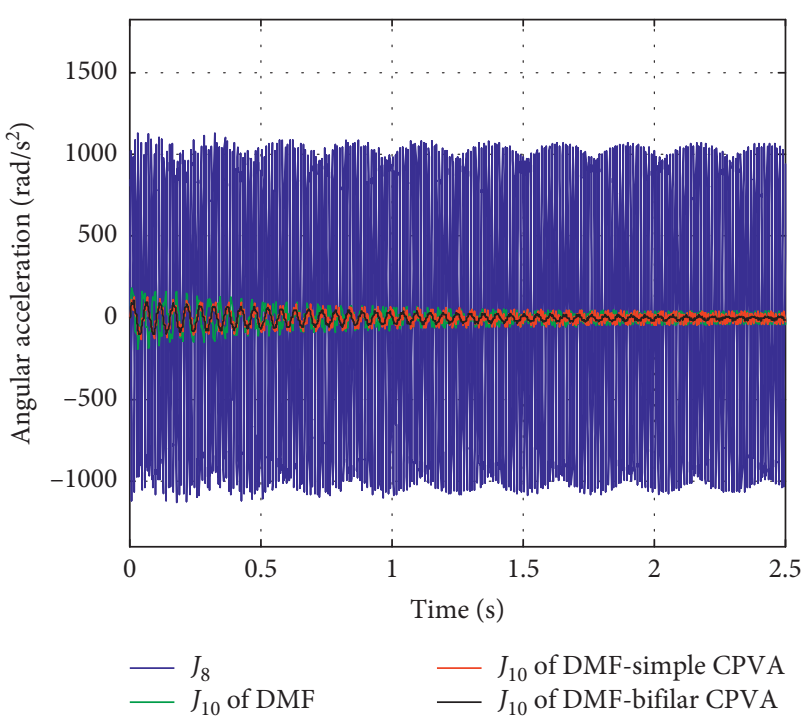

Figure 12: Angular acceleration curves of $J_{8}$ and $J_{10}$ at $3000 \mathrm{r} / \mathrm{min}$ of engine speed.

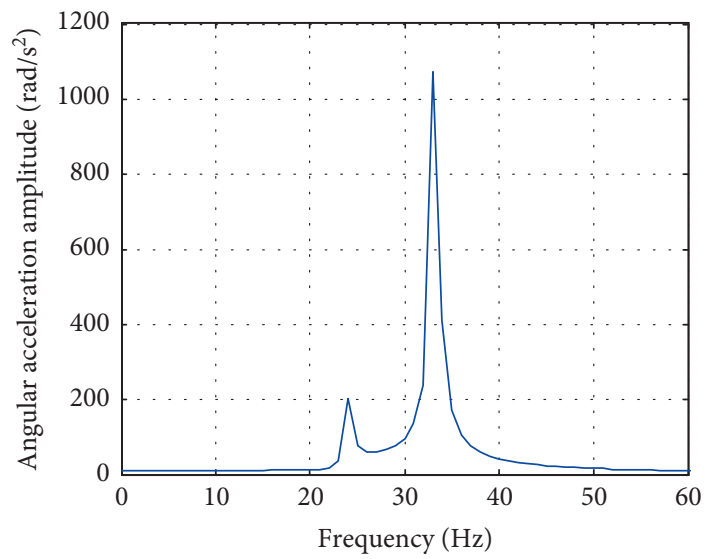

(a)

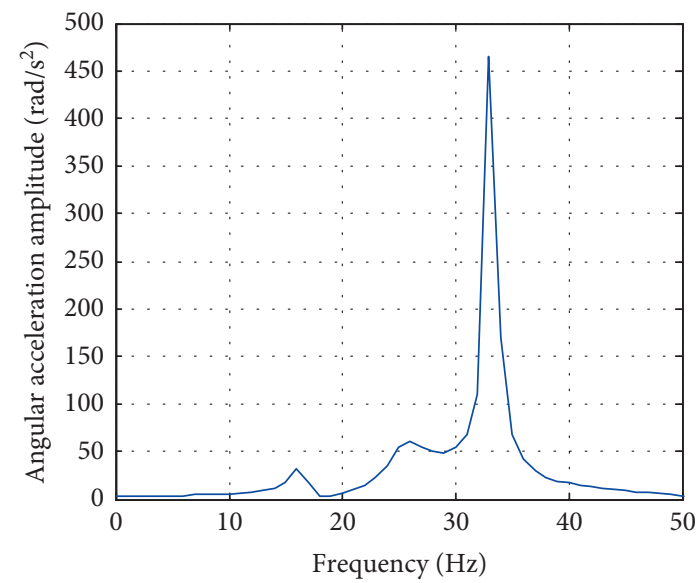

(c)

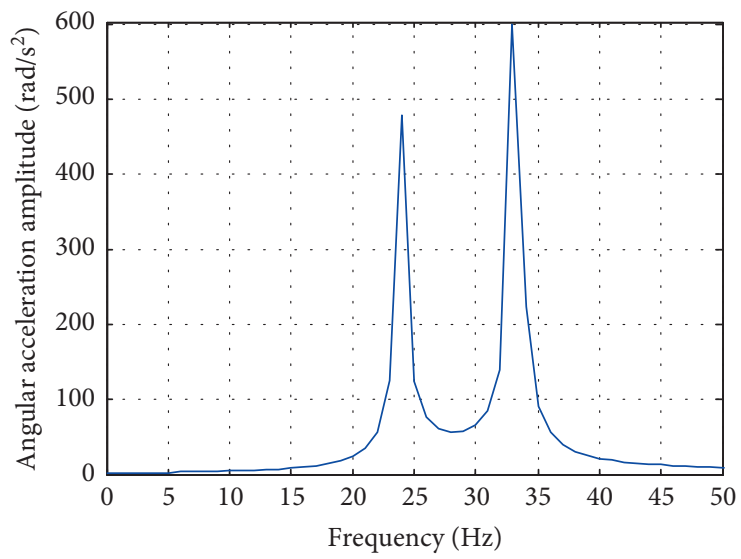

(b)

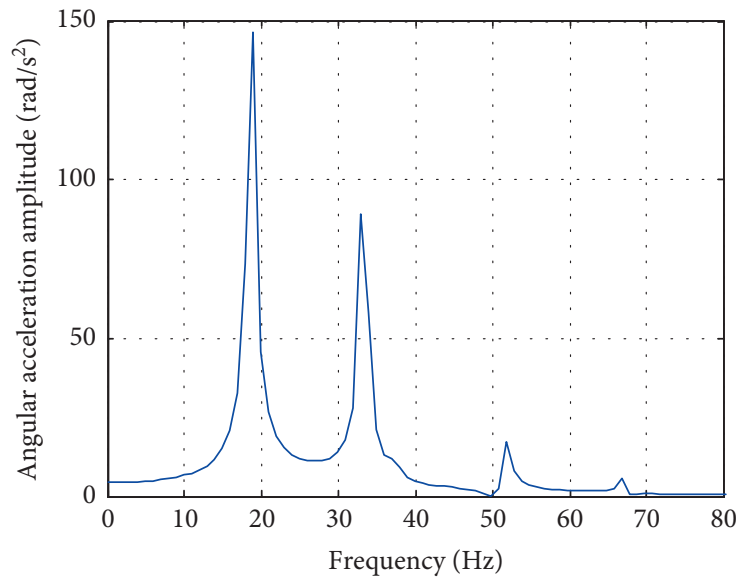

(d)

FIGURE 13: Spectra of $J_{8}$ and $J_{10}$ at $1000 \mathrm{r} / \mathrm{min}$ of engine speed: (a) spectrum of $J_{8}$ and (b) spectrum of $J_{10}$ of DMF; (c) spectrum of $J_{10}$ of DMF with simple CPVA; (d) spectrum of $J_{10}$ of DMF with bifilar CPVA. 


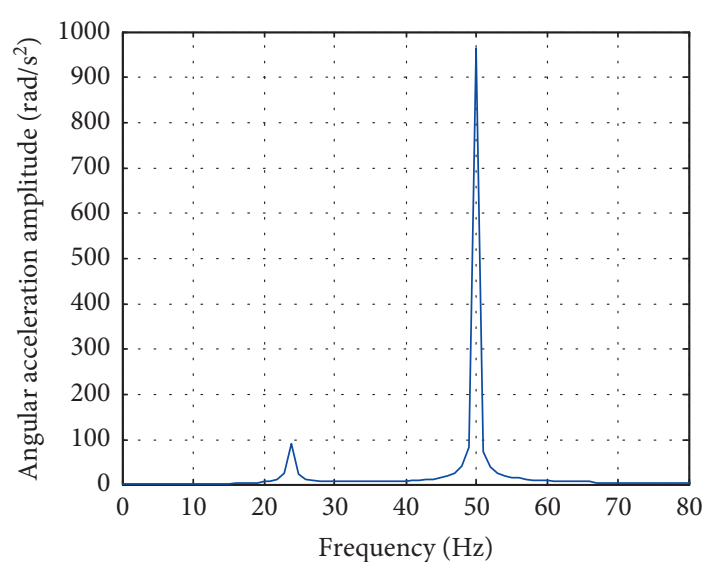

(a)

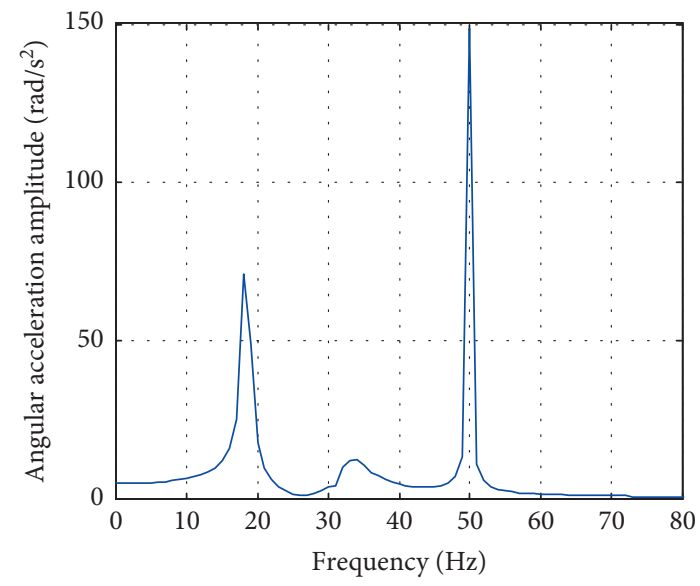

(c)

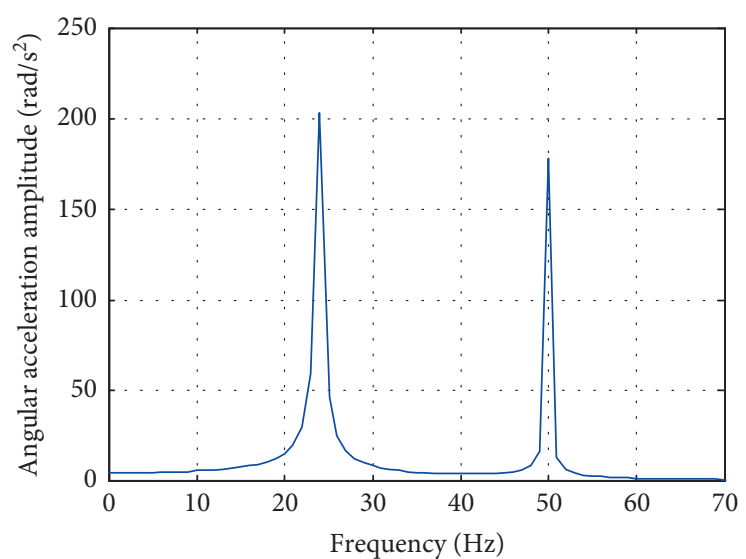

(b)

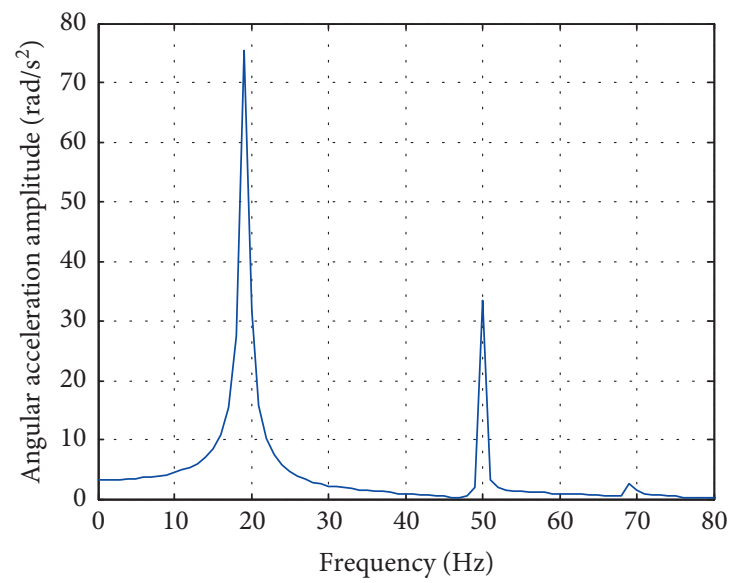

(d)

FIGURE 14: Spectra of $J_{8}$ and $J_{10}$ at $1500 \mathrm{r} / \mathrm{min}$ of engine speed: (a)spectrum of $J_{8}$ and (b) spectrum of $J 10$ of DMF; (c) spectrum of J10 of DMF with simple CPVA; (d) spectrum of $J 10$ of DMF with bifilar CPVA.

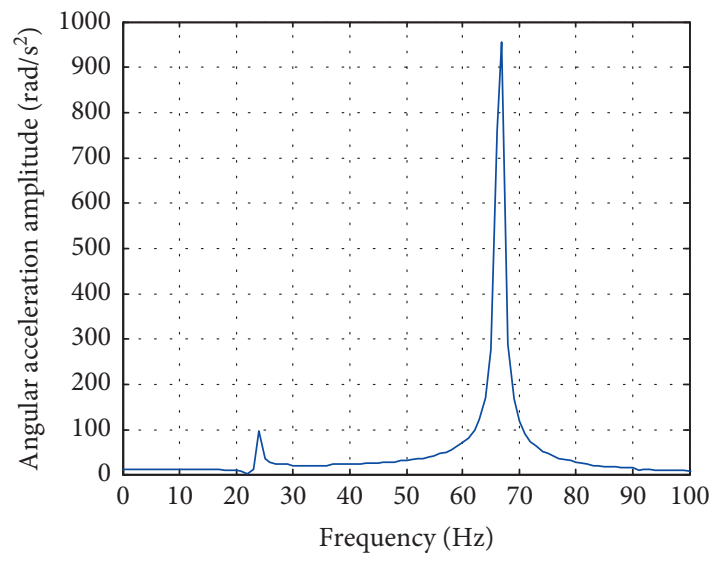

(a)

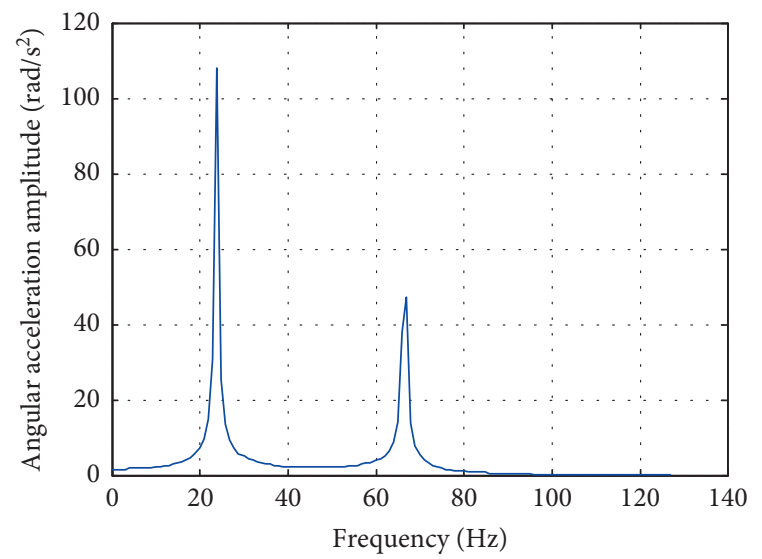

(b)

FIGURE 15: Continued. 


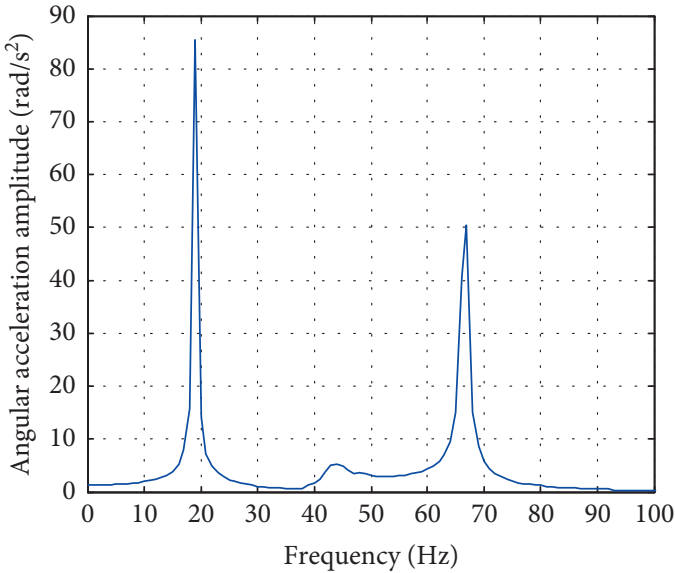

(c)

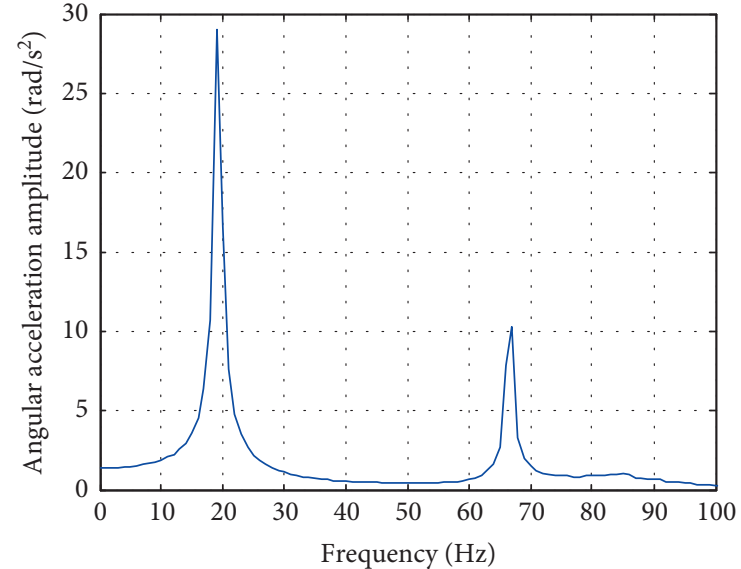

(d)

FIGURE 15: Spectra of $J_{8}$ and $J_{10}$ at $2000 \mathrm{r} / \mathrm{min}$ of engine speed: (a) spectrum of $J_{8}$ and (b) spectrum of $J_{10}$ of DMF; (c) spectrum of $J_{10}$ of DMF with simple CPVA; (d) spectrum of $J_{10}$ of DMF with bifilar CPVA.

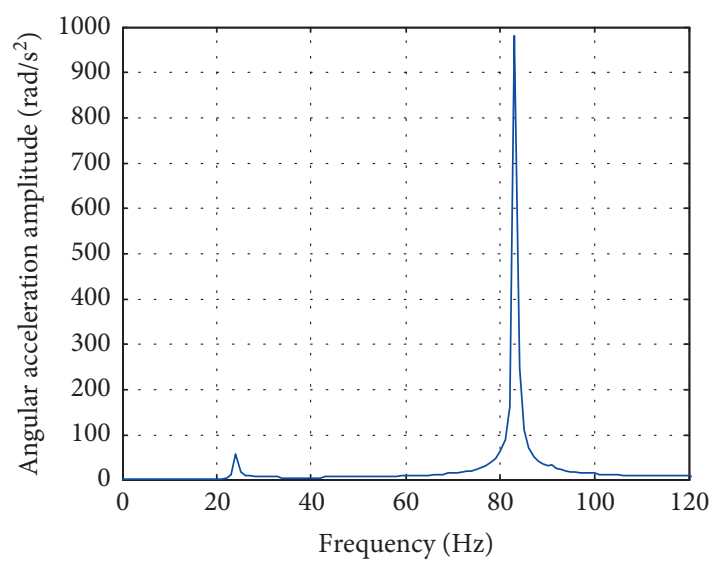

(a)

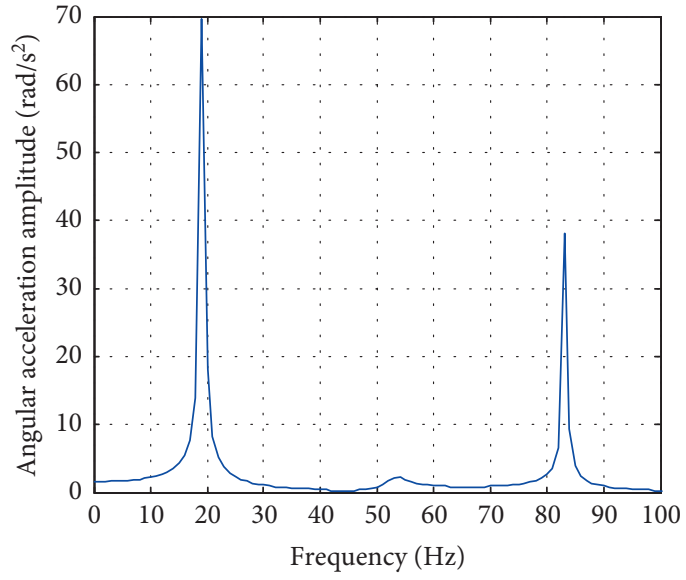

(c)

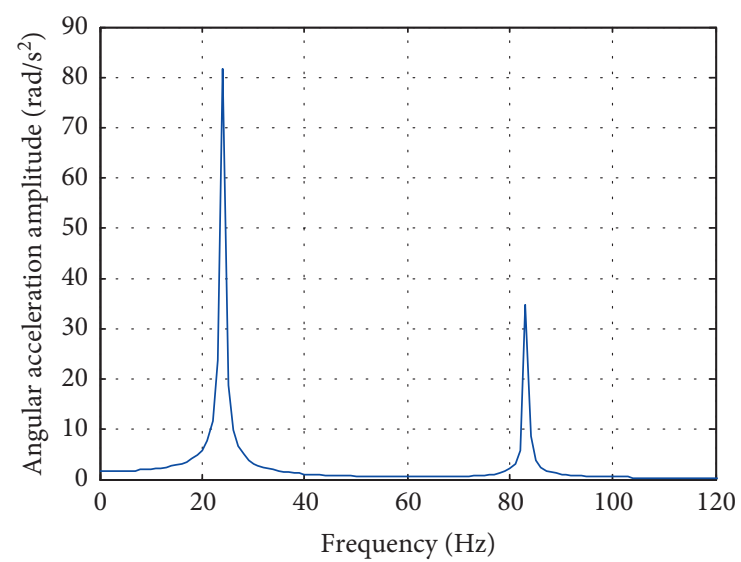

(b)

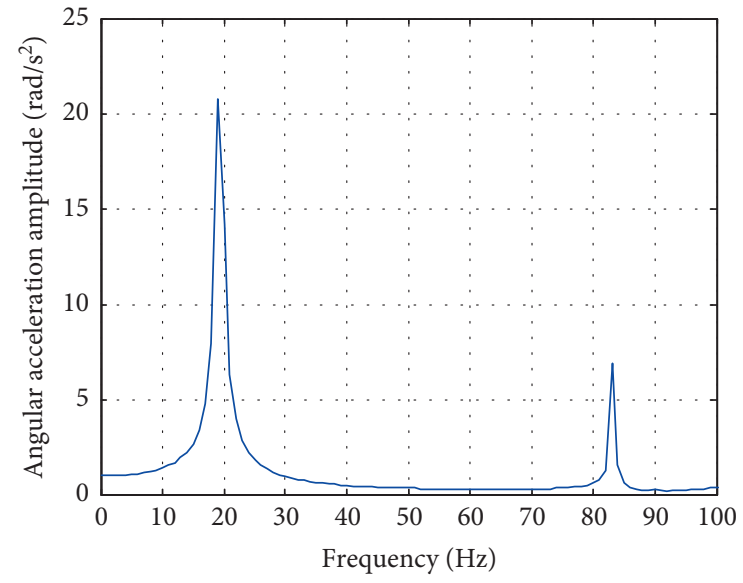

(d)

FIGURE 16: Spectra of $J_{8}$ and $J_{10}$ at $2500 \mathrm{r} / \mathrm{min}$ of engine speed: (a) spectrum of $J_{8}$ and (b) spectrum of $J_{10}$ of DMF; (c) spectrum of $J_{10}$ of DMF with simple CPVA; (d) spectrum of $J_{10}$ of DMF with bifilar CPVA. 


$$
T=m(R+l) \mu^{2} R \phi .
$$
get

By substituting equation (11) into equation (16), we can

$$
T=-\frac{m(R+l)^{2}}{1-(\omega / \mu) 2(l / R)} \ddot{\alpha} .
$$

According to equation (17), the equivalent moment of inertia of the bifilar CPVA $J_{\mathrm{e}}$ is shown as

$$
J_{e}=-\frac{m(R+l)^{2}}{1-(\omega / \mu) 2(l / R)} .
$$

Let the excitation harmonic order of the engine be $\varepsilon$, then

$$
\frac{\omega}{\mu}=\varepsilon
$$

If

$$
1-\left(\frac{\omega}{\mu}\right)^{2} \frac{l}{R}=0
$$

then $J_{e}=\infty$. In this case, $\varepsilon$ meets the following condition:

$$
\varepsilon=\sqrt{\frac{R}{l}}
$$

Based on the above discussion, the natural frequency of the bifilar CPVA is related to the engine speed, which means that the bifilar CPVA can attenuate the torsional vibration in full speed range of engine. When $\sqrt{(R / l)}$ is equal to $\varepsilon$ by adjusting the ratio of $R$ and $l$, the bifilar CPVA is equivalent to a flywheel with infinite moment of inertia; that is, the $\varepsilon^{\text {th }}$ harmonic-order torque fluctuation from the engine can be theoretically eliminated completely by the bifilar CPVA.

\section{Nonlinear Dynamic Model of DMF with a Bifilar CPVA}

3.1. Nonlinear Dynamic Model of DMF. The mechanical model of the DMF with a bifilar CPVA is shown in Figure 3, in which the moment of inertia and the angular displacement of the primary flywheel assembly are $J_{\mathrm{p}}$ and $\beta$, respectively; the moment of inertia and the angular displacement of the secondary flywheel assembly are $J_{\mathrm{s}}$ and $\alpha$, respectively; the moment of inertia of the bifilar CPVA is $I$; the torsional stiffness of the DMF is $K$; and the damping coefficient of the bifilar CPVA is $C_{a}$.

During the operation of the DMF, the friction between the spring and slide contains Coulomb friction and viscous friction, which characterizes hysteresis nonlinearity [10]. The author of this paper has created the nonlinear dynamic model of the DMF [24], in which the improved Bouc-Wen model was used to describe the nonlinear hysteresis torque. The dynamic equations of the DMF have been deduced as follows:

$$
\left[\begin{array}{cc}
J_{p} & 0 \\
0 & J_{s}
\end{array}\right]\left[\begin{array}{l}
\ddot{\beta} \\
\ddot{\alpha}
\end{array}\right]+\left[\begin{array}{cc}
K & -K \\
-K & K
\end{array}\right]\left[\begin{array}{l}
\beta \\
\alpha
\end{array}\right]+\left[\begin{array}{c}
Z(\gamma, \dot{\gamma}) \\
-Z(\gamma, \dot{\gamma})
\end{array}\right]=\left[\begin{array}{l}
T \\
0
\end{array}\right]
$$

$$
\begin{gathered}
\gamma(t)=\beta(t)-\alpha(t), \\
Z(\gamma, \dot{\gamma})=Z_{r d}(\dot{\gamma})+Z_{b w}(\gamma, \dot{\gamma}), \\
Z_{r d}(\dot{\gamma})=C_{s} \cdot|\dot{\gamma}|^{b} \cdot \operatorname{sign}(\dot{\gamma}), \\
Z_{b w}(\gamma, \dot{\gamma})=\eta \cdot \dot{\gamma}(t)-\lambda \cdot|\dot{\gamma}(t)| \cdot Z_{b w}(\gamma, \dot{\gamma}) \\
\cdot\left|Z_{b w}(\gamma, \dot{\gamma})\right|^{c-1}-\mu \cdot \dot{\gamma}(t) \cdot\left|Z_{b w}(\gamma, \dot{\gamma})\right|^{c},
\end{gathered}
$$

where $\mu, \lambda, \eta, c$, and $b$ are the Bouc-Wen model parameters to be determined, $Z(\gamma, \dot{\gamma})$ is the frictional torque in the DMF, and $T$ is the input torque of the primary flywheel assembly. On the basis of the model, $Z(\gamma, \dot{\gamma})$ can be given by

$$
Z(\gamma, \dot{\gamma})=\frac{1}{2}\left(T-J_{p} \cdot \ddot{\beta}+J_{s} \cdot \ddot{\alpha}\right)-K(\beta-\alpha) .
$$

Thus, the parameters of the Bouc-Wen model can be identified based on the dynamic test data, and the identification method has been described in the literature [20].

3.2. Nonlinear Dynamic Model of the DMF with Biflar CPVA. Referring to Figure 2, the coordinates of $M\left(x_{M}, y_{M}\right)$ are expressed as

$$
\begin{aligned}
& x_{M}=R \cos (\alpha)+l \cos (\alpha+\phi), \\
& y_{M}=R \sin (\alpha)+l \sin (\alpha+\phi) .
\end{aligned}
$$

Then,

$$
\begin{aligned}
& \dot{x}_{M}=R \dot{\alpha} \sin (\alpha)-l(\dot{\alpha}+\dot{\phi}) \sin (\alpha+\phi), \\
& \dot{y}_{M}=R \dot{\alpha} \cos (\alpha)+l(\dot{\alpha}+\dot{\phi}) \cos (\alpha+\phi) .
\end{aligned}
$$

Thus, the velocity of $M$ can be obtained from the following equation:

$$
\left|v_{M}\right|^{2}=\left(\dot{x}_{M}\right)^{2}+\left(\dot{y}_{M}\right)^{2}=R^{2} \dot{\alpha}^{2}+2 R l \dot{\alpha}(\dot{\alpha}+\dot{\phi}) \cos (\phi)+l^{2}(\dot{\phi}+\dot{\alpha})^{2} .
$$

The kinetic energy $U$ of the DMF with the bifilar CPVA can be written as

$$
\begin{aligned}
U= & \frac{1}{2} J_{p} \beta^{2}+\frac{1}{2}\left(J_{s}+I\right) \dot{\alpha}^{2}+\frac{1}{2} m\left|v_{M}\right|^{2} \\
= & \frac{1}{2} J_{p} \beta^{2}+\frac{1}{2}(J+I) \dot{\alpha}^{2}+\frac{1}{2} m R^{2} \dot{\alpha}^{2} \\
& +m R l \dot{\alpha}(\dot{\alpha}+\dot{\phi}) \cos (\phi)+\frac{1}{2} m l^{2}(\dot{\phi}+\dot{\alpha})^{2} .
\end{aligned}
$$

Since the gravitational potential energy of the system is too small compared with the elastic potential energy, the potential energy $\mathrm{V}$ can be given by 


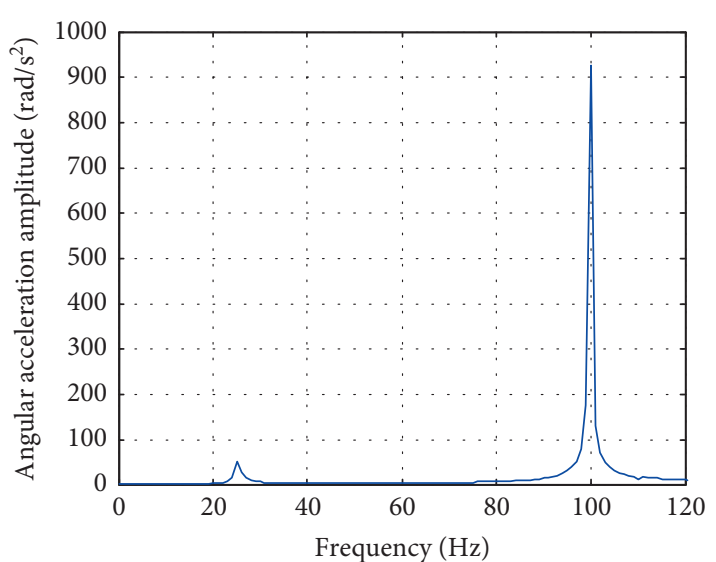

(a)

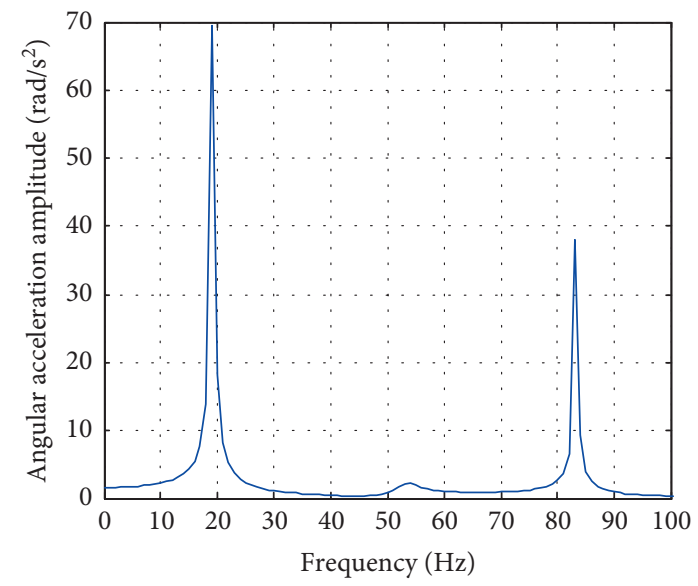

(c)

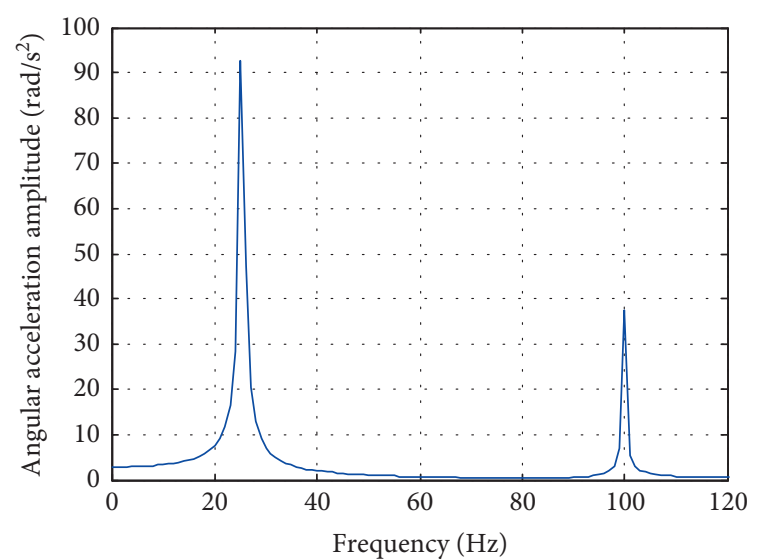

(b)

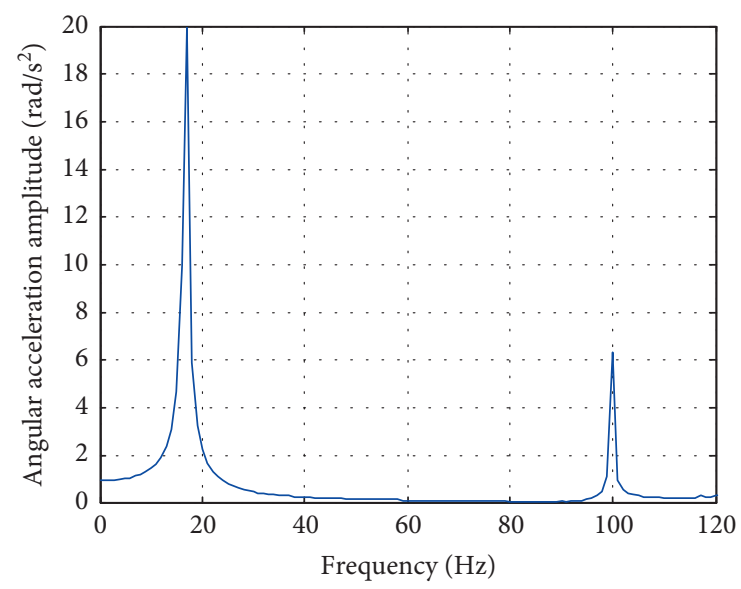

(d)

FIGURE 17: Spectra of $J_{8}$ and $J_{10}$ at $3000 \mathrm{r} / \mathrm{min}$ of engine speed: (a) spectrum of $J_{8}$ and (b) spectrum of $J_{10}$ of DMF; (c) spectrum of $J_{10}$ of DMF with simple CPVA; (d) spectrum of $J_{10}$ of DMF with bifilar CPVA.

TABLE 4: The overall angular acceleration amplitudes corresponding to different rotational speeds.

\begin{tabular}{lcccc}
\hline Engine speed $(\mathrm{r} / \mathrm{min})$ & $J_{8}\left(\mathrm{rad} / \mathrm{s}^{2}\right)$ & $\mathrm{DMF} J_{10}\left(\mathrm{rad} / \mathrm{s}^{2}\right)$ & DMF with simple CPVA $J_{10}\left(\mathrm{rad} / \mathrm{s}^{2}\right)$ & DMF with bifilar CPVA $\left(\mathrm{rad} / \mathrm{s}^{2}\right)$ \\
\hline 800 & 210.0 & 90.00 & 53.00 & 30 \\
1000 & 1350 & 800.0 & 495.0 & 152.0 \\
1500 & 1065 & 250.0 & 165.0 & 80.00 \\
2000 & 1030 & 115.00 & 92.00 & 31.00 \\
2500 & 1030 & 95.00 & 85.00 & 23.00 \\
3000 & 1030 & 96.00 & 85.00 & 21.00 \\
\hline
\end{tabular}

TABLE 5: The $2^{\text {nd }}$ order angular acceleration amplitudes corresponding to different rotational speeds.

\begin{tabular}{lcccc}
\hline Engine speed $(\mathrm{r} / \mathrm{min})$ & $J_{8}\left(\mathrm{rad} / \mathrm{s}^{2}\right)$ & $\mathrm{DMF} J_{10}\left(\mathrm{rad} / \mathrm{s}^{2}\right)$ & DMF with simple CPVA $J_{10}\left(\mathrm{rad} / \mathrm{s}^{2}\right)$ & DMF with bifilar CPVA $\left(\mathrm{rad} / \mathrm{s}^{2}\right)$ \\
\hline 800 & 170 & 70.00 & 42.00 & 26 \\
1000 & 1100 & 600.0 & 465.0 & 80.0 \\
1500 & 980 & 175.0 & 150.0 & 35.00 \\
2000 & 950 & 50.00 & 50.00 & 12.00 \\
2500 & 960 & 36.00 & 37.00 & 8.00 \\
3000 & 930 & 37.00 & 37.00 & 7.00 \\
\hline
\end{tabular}




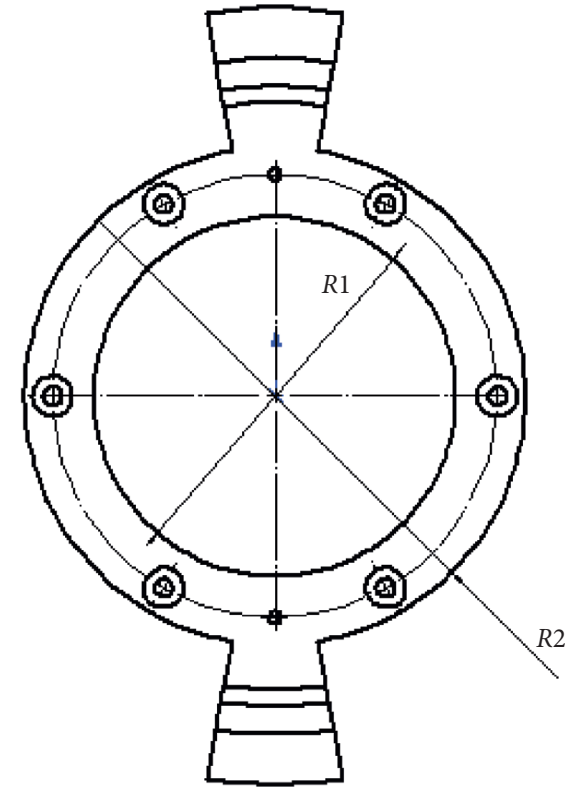

Figure 18: Structure diagram of the driven plate.

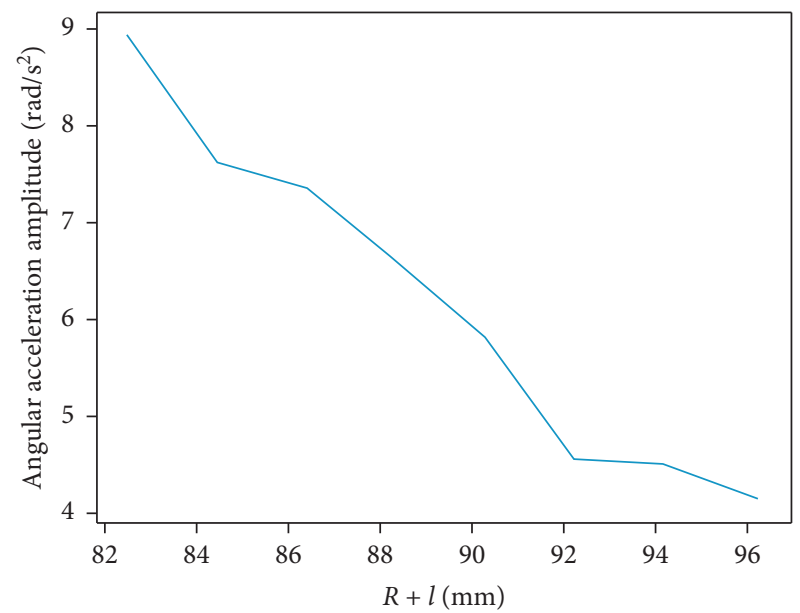

FIGURE 19: Variation in angular acceleration amplitude of $J_{10}$ with $R+l$.

$$
V=\frac{1}{2} k(\beta-\alpha)^{2}
$$

Then, the Lagrangian $L$ of the system is described as

$$
L=U-V=\frac{1}{2} J_{p} \dot{\beta}^{2}+\frac{1}{2}\left(J_{s}+I\right) \dot{\alpha}^{2}+\frac{1}{2} m R^{2} \dot{\alpha}^{2}
$$

$m R l \dot{\alpha}(\dot{\alpha}+\dot{\phi}) \cos (\phi)+\frac{1}{2} m l^{2}(\dot{\phi}+\dot{\alpha})^{2}-\frac{1}{2} k(\beta-\alpha)^{2}$.

For the primary flywheel assembly, the generalized force $M_{1}$ is given by

$$
M_{1}=T-Z(\gamma, \dot{\gamma})
$$

For the secondary flywheel assembly, the generalized force $M_{2}$ is described as

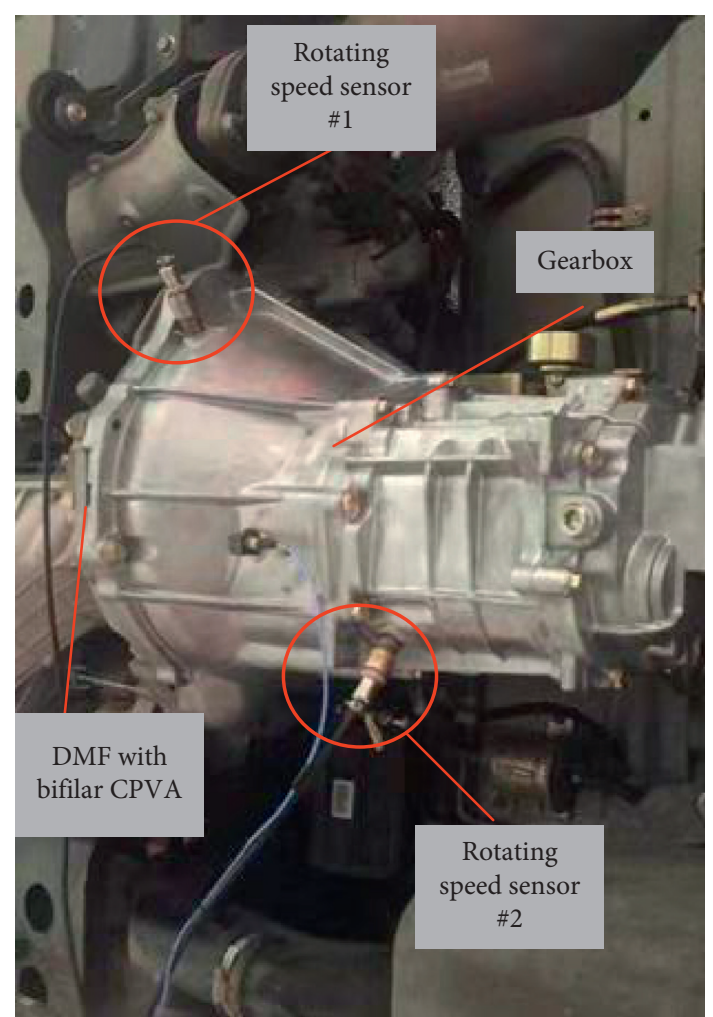

Figure 20: Sensors layout of real vehicle test.

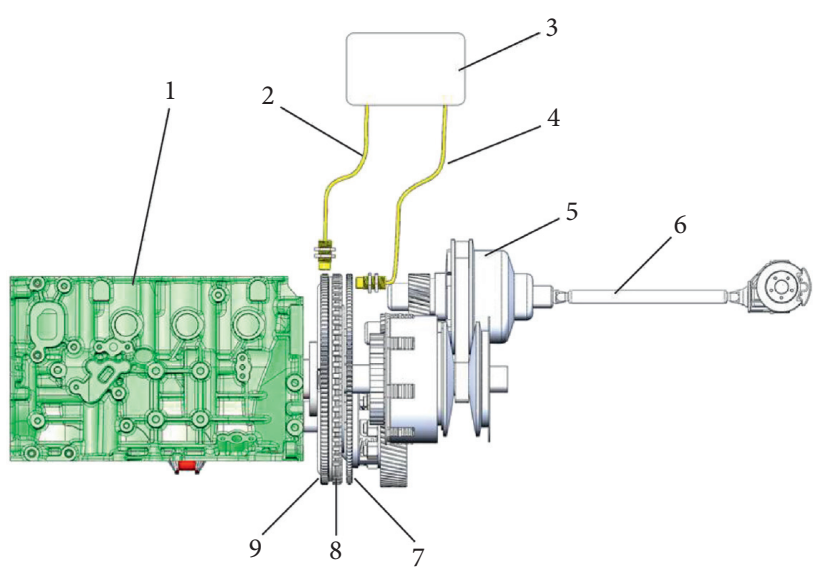

FIGURE 21: Schematic diagram of electromagnetic rotating speed sensors arrangement. 1 , engine; 2 , the no. 1 sensor; 3 , Siemens data acquisition instrument; 4, the no. 2 sensor; 5, CVT gearbox; 6, transmission shaft; 7 , the signal gear on the input shaft of the gearbox; 8, DMF; 9, the signal gear on the primary flywheel.

$$
M_{2}=Z(\gamma, \dot{\gamma})
$$

For the bifilar CPVA, the generalized force $M_{3}$ is shown as

$$
M_{3}=-C_{a} \dot{\phi}
$$

Using Lagrangian mechanics, the Lagrangian equations of motion are expressed as 


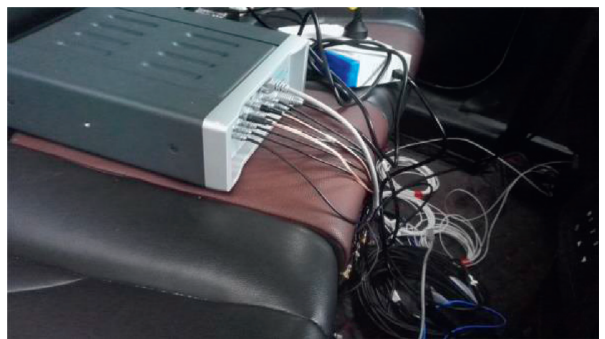

FIgURE 22: Digital acquisition hardware inside the car.

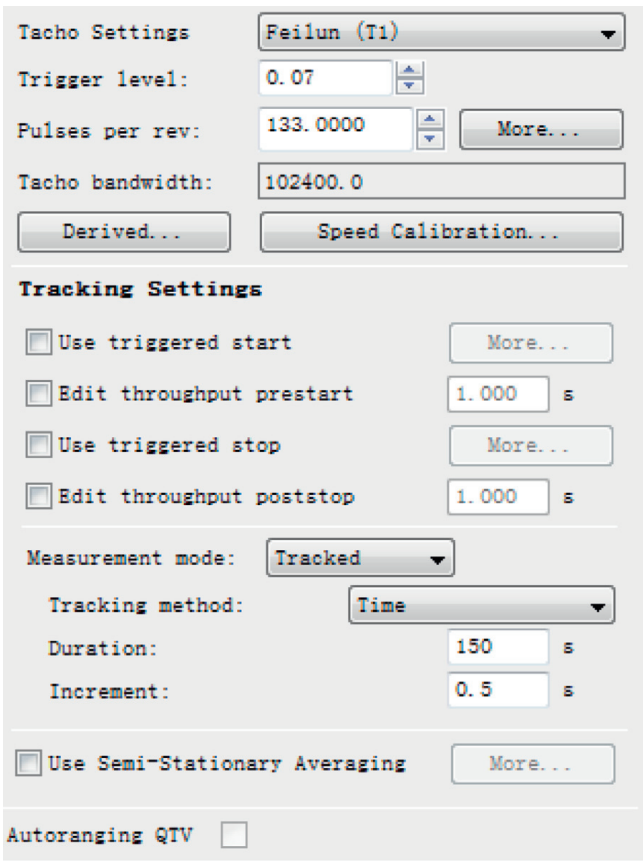

(a)

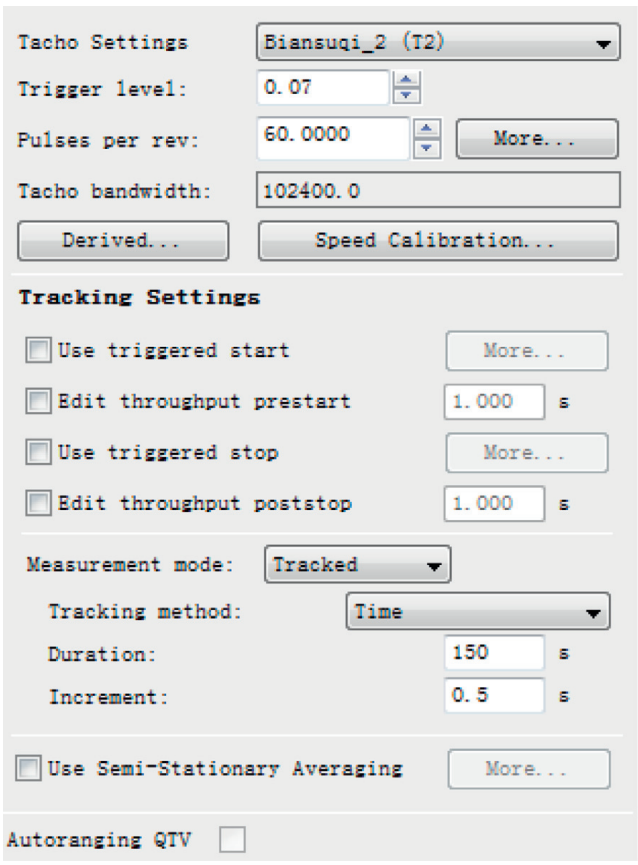

(b)

FIGURE 23: Measurement tracking setting: (a) tracking setting of rotating speed signal channel of the primary flywheel; (b) tracking setting of rotating speed signal channel of the input shaft of the gearbox.

$$
\begin{aligned}
& \frac{d}{d t}\left(\frac{\partial L}{\partial \dot{\beta}}\right)-\frac{\partial L}{\partial \beta}=M_{1}, \\
& \frac{d}{d t}\left(\frac{\partial L}{\partial \dot{\alpha}}\right)-\frac{\partial L}{\partial \alpha}=M_{2}, \\
& \frac{d}{d t}\left(\frac{\partial L}{\partial \dot{\phi}}\right)-\frac{\partial L}{\partial \phi}=M_{3} .
\end{aligned}
$$

Substituting equation (35) into equations (39), (40), and (41), the nonlinear dynamic model of the DMF with bifilar CPVA can be obtained as

$$
\begin{gathered}
J_{p} \ddot{\beta}+K(\beta-\alpha)=T-Z(\gamma, \dot{\gamma}), \\
\left(J_{s}+m\left(l^{2}+R^{2}\right)+2 m R l \cos (\phi)+I\right) \ddot{\alpha} \\
+m\left(l^{2}+R l \cos (\phi)\right) \ddot{\phi}-m R l \sin (\phi) \dot{\phi}^{2} \\
-2 m R l \sin (\phi) \dot{\alpha} \dot{\phi}+k(\alpha-\beta)=Z(\gamma, \dot{\gamma}),
\end{gathered}
$$

$$
m\left(l^{2}+R l \cos (\phi)\right) \ddot{\alpha}+m l^{2} \ddot{\phi}+m R l \sin (\phi) \dot{\phi}^{2}=-C_{a} \dot{\phi}
$$

3.3. Dynamic Model of Power Transmission System. Referring to the structural parameters of a certain vehicle with a four-cylinder and four-stroke engine, the torsional vibration models of 10 degrees of freedom and 11 degrees of freedom of the power transmission system with the DMF with bifilar CPVA are developed, respectively, under idling and driving conditions, as depicted in Figures 4 and 5 .

In Figures 4 and $5, J_{i}$ is the moment of inertia of each rotating element of the vehicle powertrain, $K_{\mathrm{i}}$ is the torsional stiffness of each elastic element, and $C_{a}$ is the viscous damping coefficient between the bifilar CPVA and the secondary flywheel assembly. The specific meanings and values of these parameters are listed in Tables 1 and 2 . The equations of motion of the power transmission system are deduced as follows: 


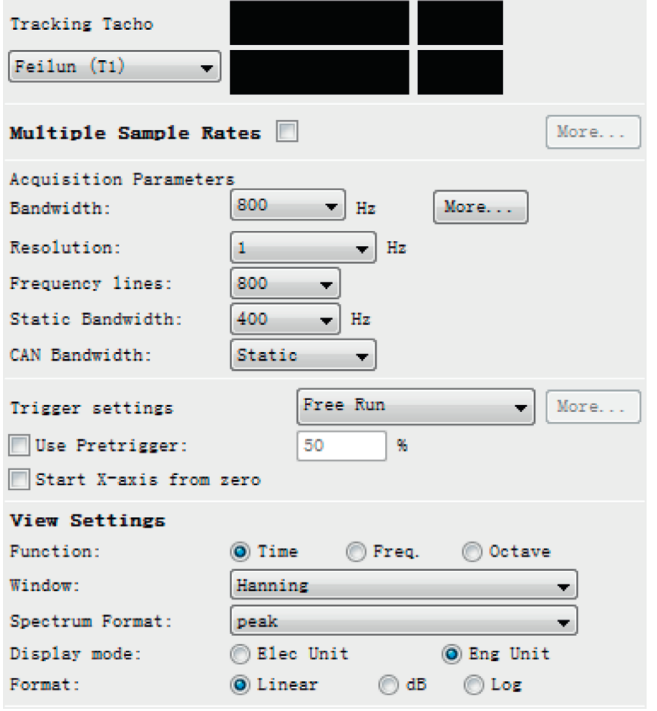

(a)

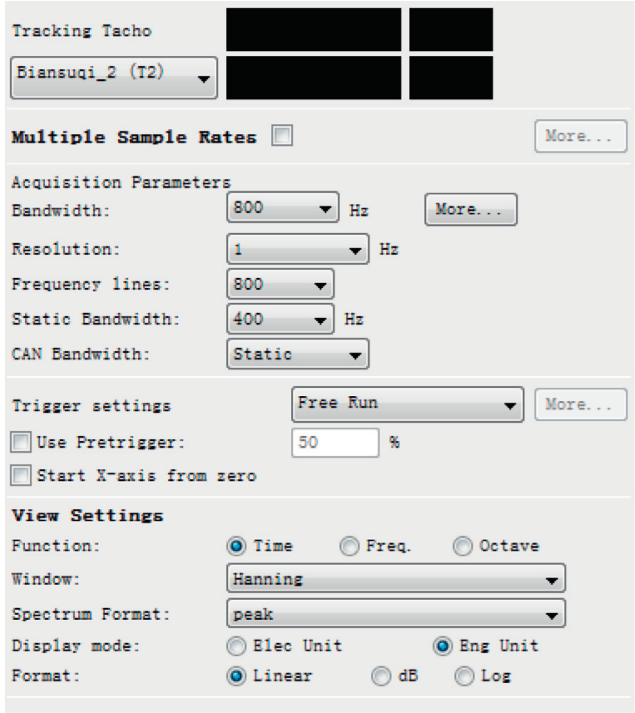

(b)

FIGURE 24: Acquisition setup: (a) acquisition setting of rotating speed signal channel of the primary flywheel; (b) acquisition setting of rotating speed signal channel of the input shaft of the gearbox.

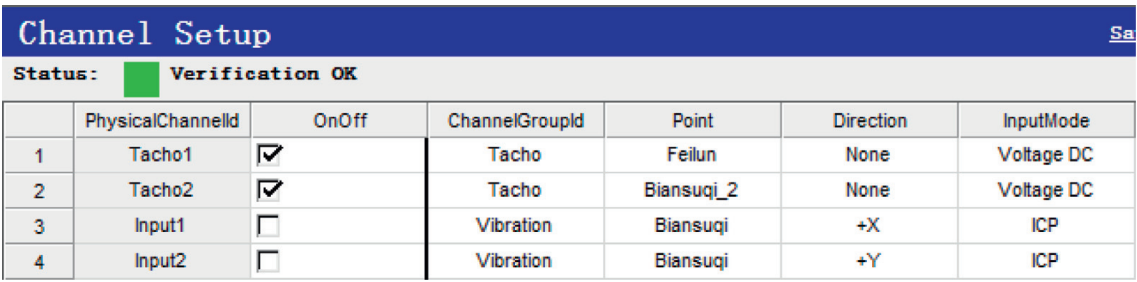

FIGURE 25: Measurement channels setting.

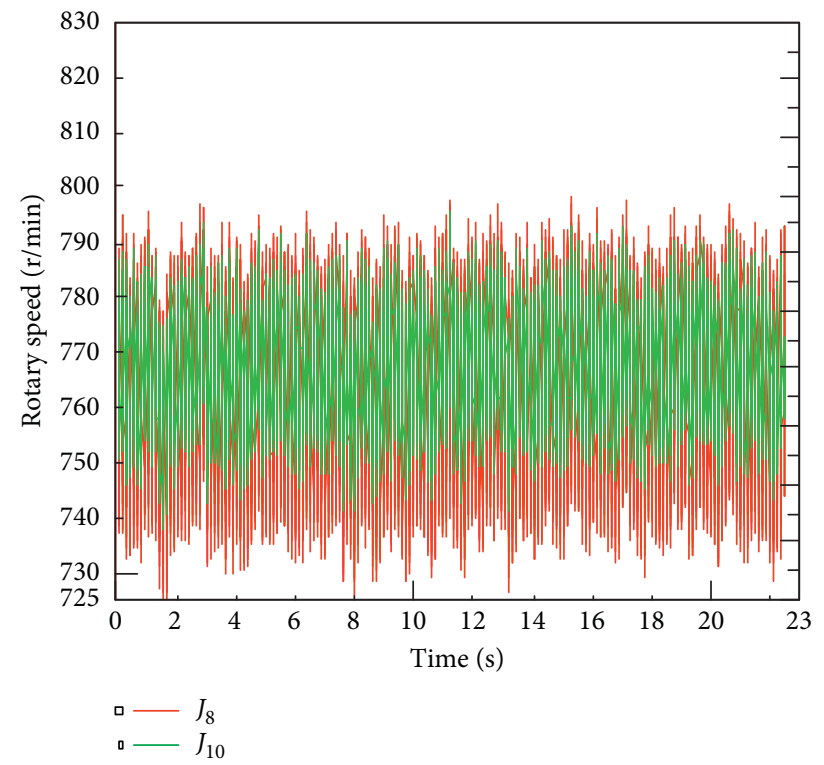

FIGURE 26: The speed curves of the primary flywheel and the input shaft of the gearbox matching the DMF under the idling condition. 


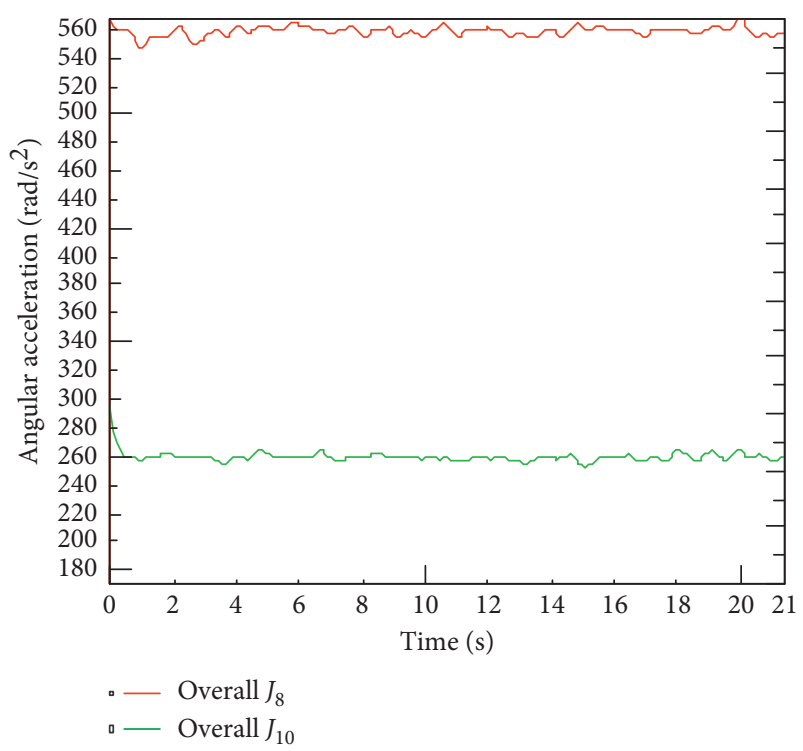

FIgURE 27: The overall angular acceleration curves of the primary flywheel and the input shaft of the gearbox matching the DMF under the idling condition.

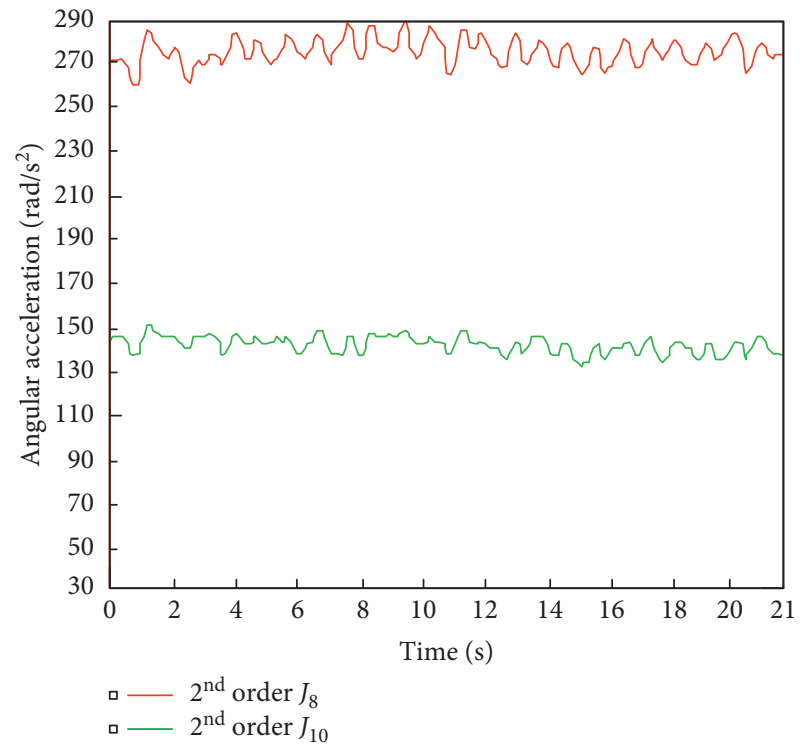

FIGURE 28: The $2^{\text {nd }}$ order angular acceleration curves of the primary flywheel and the input shaft of the gearbox matching the DMF under the idling condition.

TABLE 6: Angular acceleration amplitudes under the idling condition for the powertrain with the DMF.

\begin{tabular}{lcc}
\hline Items & $J_{8}\left(\mathrm{rad} / \mathrm{s}^{2}\right)$ & $J_{10}\left(\mathrm{rad} / \mathrm{s}^{2}\right)$ \\
\hline The overall angular acceleration & 550 & 260 \\
The $2^{\text {nd }}$ order angular acceleration & 280 & 140 \\
\hline
\end{tabular}

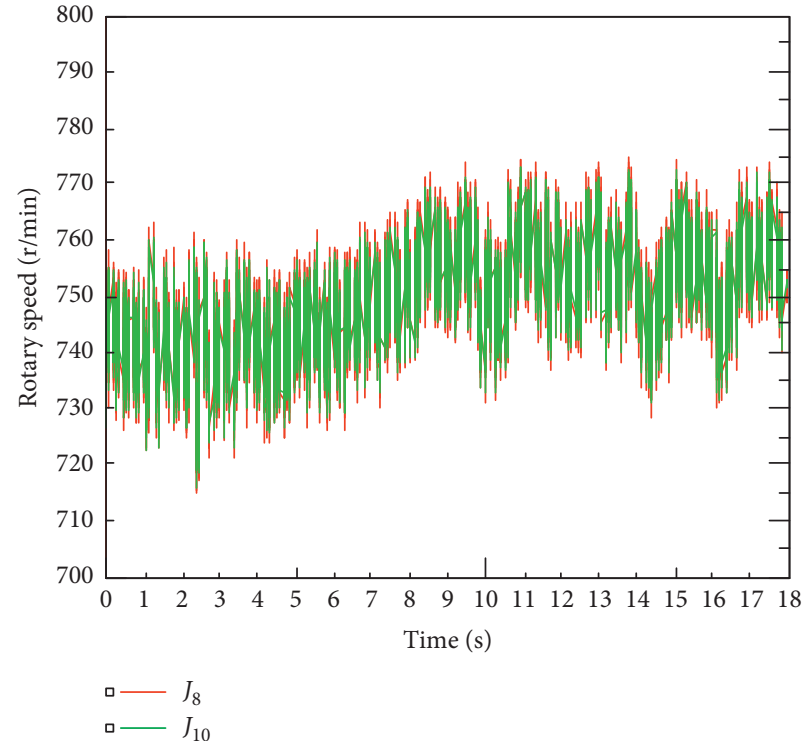

FIGURE 29: The rotary speed curves of the primary flywheel and the input shaft of the gearbox matching the DMF with the bifilar CPVA under the idling condition.

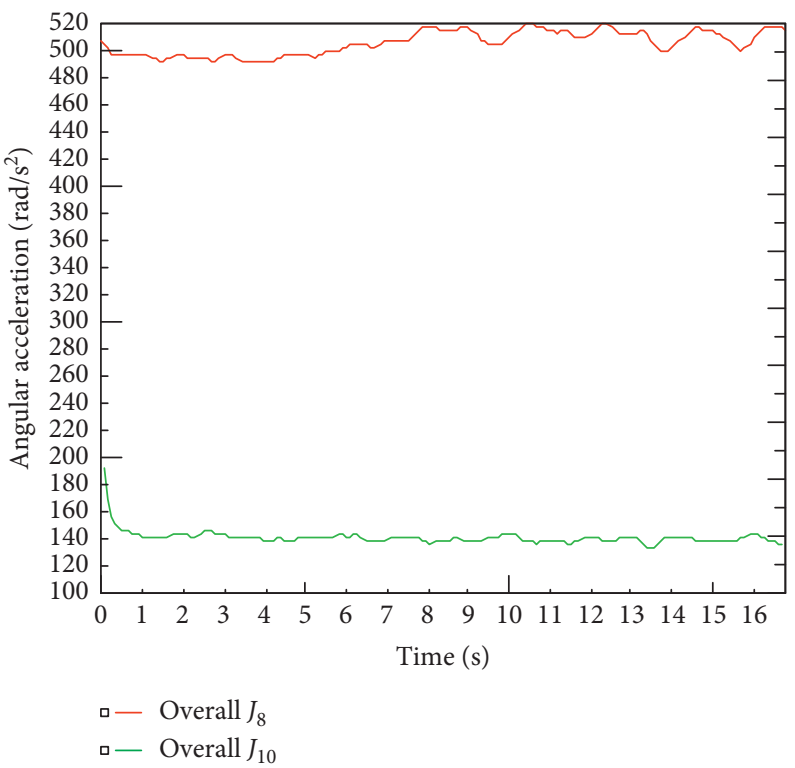

FIGURE 30: The overall angular acceleration curves of the primary flywheel and the input shaft of the gearbox matching the DMF with the bifilar CPVA under the idling condition. 


$$
\begin{aligned}
& \left\{\begin{array}{l}
J_{1} \ddot{\alpha}_{1}+k_{1}\left(\alpha_{1}-\alpha_{2}\right)=0 \\
J_{2} \ddot{\alpha}_{2}-k_{1}\left(\alpha_{1}-\alpha_{2}\right)+k_{2}\left(\alpha_{2}-\alpha_{3}\right)=0 \\
J_{3} \ddot{\alpha}_{3}-k_{2}\left(\alpha_{2}-\alpha_{3}\right)+k_{3}\left(\alpha_{3}-\alpha_{4}\right)=0 \\
J_{4} \ddot{\alpha}_{4}-k_{3}\left(\alpha_{3}-\alpha_{4}\right)+k_{4}\left(\alpha_{4}-\alpha_{5}\right)=T_{1} \\
J_{5} \ddot{\alpha}_{5}-k_{4}\left(\alpha_{4}-\alpha_{5}\right)+k_{5}\left(\alpha_{5}-\alpha_{6}\right)=T_{2} \\
J_{6} \ddot{\alpha}_{6}-k_{5}\left(\alpha_{5}-\alpha_{6}\right)+k_{6}\left(\alpha_{6}-\alpha_{7}\right)=T_{3} \\
J_{7} \ddot{\alpha}_{7}-k_{6}\left(\alpha_{6}-\alpha_{7}\right)+k_{7}\left(\alpha_{7}-\alpha_{8}\right)=T_{4} \\
J_{8} \ddot{\alpha}_{8}-k_{7}\left(\alpha_{7}-\alpha_{8}\right)+k_{8}\left(\alpha_{8}-\alpha_{9}\right)=-Z(\gamma, \dot{\gamma}) \\
J_{9}+I+m\left(l^{2}+R^{2}\right)+2 m R l \cos (\phi) \alpha_{9}+\left(m l^{2}+m R l \cos (\ddot{\phi})\right) \ddot{\phi}-m R l \sin (\phi) \dot{\phi}^{2} \\
-2 m R l \sin (\phi) \dot{\alpha}_{9} \dot{\phi}+k_{8}\left(\alpha_{9}-\alpha_{8}\right)+k_{9}\left(\alpha_{9}-\alpha_{10}\right)=Z(\gamma, \dot{\gamma}) \\
\left(m l^{2}+m R l \cos (\phi)\right) \ddot{\alpha}_{9}+m l^{2} \ddot{\phi}+m R l \sin (\phi) \dot{\alpha}_{9}^{2}=-C_{a} \dot{\phi} \\
J_{10} \ddot{\alpha}_{10}-k_{9}\left(\alpha_{9}-\alpha_{10}\right)=0,
\end{array}\right. \\
& \left\{\begin{array}{l}
J_{1} \ddot{\alpha}_{1}+k_{1}\left(\alpha_{1}-\alpha_{2}\right)=0 \\
J_{2} \ddot{\alpha}_{2}-k_{1}\left(\alpha_{1}-\alpha_{2}\right)+k_{2}\left(\alpha_{2}-\alpha_{3}\right)=0 \\
J_{3} \ddot{\alpha}_{3}-k_{2}\left(\alpha_{2}-\alpha_{3}\right)+k_{3}\left(\alpha_{3}-\alpha_{4}\right)=0 \\
J_{4} \ddot{\alpha}_{4}-k_{3}\left(\alpha_{3}-\alpha_{4}\right)+k_{4}\left(\alpha_{4}-\alpha_{5}\right)=T_{1} \\
J_{5} \ddot{\alpha}_{5}-k_{4}\left(\alpha_{4}-\alpha_{5}\right)+k_{5}\left(\alpha_{5}-\alpha_{6}\right)=T_{2} \\
J_{6} \ddot{\alpha}_{6}-k_{5}\left(\alpha_{5}-\alpha_{6}\right)+k_{6}\left(\alpha_{6}-\alpha_{7}\right)=T_{3} \\
J_{7} \ddot{\alpha}_{7}-k_{6}\left(\alpha_{6}-\alpha_{7}\right)+k_{7}\left(\alpha_{7}-\alpha_{8}\right)=T_{4} \\
J_{8} \ddot{\alpha}_{8}-k_{7}\left(\alpha_{7}-\alpha_{8}\right)+k_{8}\left(\alpha_{8}-\alpha_{9}\right)=-Z(\gamma, \dot{\gamma}) \\
J_{9}+I+m\left(l^{2}+R^{2}\right)+2 m R l \cos (\phi) \alpha_{9}+\left(m l^{2}+m R l \cos (\ddot{\phi})\right) \ddot{\phi}-m R l \sin (\phi) \dot{\phi}^{2} \\
-2 m R l \sin (\phi) \dot{\alpha}_{9} \dot{\phi}+k_{8}\left(\alpha_{9}-\alpha_{8}\right)+k_{9}\left(\alpha_{9}-\alpha_{10}\right)=Z(\gamma, \dot{\gamma}) \\
\left(m l^{2}+m R l \cos (\phi)\right) \ddot{\alpha}_{9}+m l^{2} \ddot{\phi}+m R l \sin (\phi) \dot{\alpha}_{9}^{2}=-C_{a} \dot{\phi} \\
J_{10} \ddot{\alpha}_{10}-k_{9}\left(\alpha_{9}-\alpha_{10}\right)+k_{10}\left(\alpha_{10}-\alpha_{11}\right)=0 \\
J_{11} \ddot{\alpha}_{11}-k_{10}\left(\alpha_{10}-\alpha_{11}\right)=0,
\end{array}\right.
\end{aligned}
$$

where $\alpha_{\mathrm{i}}$ is the angular displacement of each rotating element, $\phi$ is the swing angle of the bifilar CPVA, and $T_{\mathrm{i}}$ is the excitation torque acting on the crankshaft.

\section{Simulation Analysis}

The parameters of the dynamic models of the power transmission system with the DMF of the bifilar CPVA are listed in Tables 1 and 2, which are from the vehicle manufacturer Dongfeng Xiaokang Automobile Co. Ltd.

The algorithm to solve the equations of motion and simulate the dynamic behaviors is summarized in Table 3 . The purpose of the simulation is to compare the damping performance of the DMF with bifilar CPVA, the DMF with simple CPVA, and the DMF under idling and driving conditions. The amplitude of the angular acceleration of the input shaft of the gearbox $\left(J_{10}\right)$ is used as an index to predict the damping performance [1]. The dynamic model of the simple CPVA engaged in the simulation is referred to the literatures $[12,18,19]$.

In the following simulation analysis, all acceleration amplitudes refer to the half of the peak-to-peak amplitudes. In addition, we cannot obtain the actual excitation torque values of the engine from the engine manufacturer, and the excitation torque values in the following simulation under idling and driving conditions are set by the way of estimation.

4.1. Dynamic Response under the Idling Condition. Under the idling condition, the engine speed commonly is around $800 \mathrm{r} / \mathrm{min}$ and thus $\omega_{e}=800 \mathrm{r} / \mathrm{min}$. Simultaneously, the vehicle is equipped with a four-cylinder and four-stroke engine; accordingly, the main harmonic-order $\varepsilon$ of the excitation from the engine is 2 . In addition, the ignition sequence of the engine is $1,3,4$, and 2 . Therefore, the 


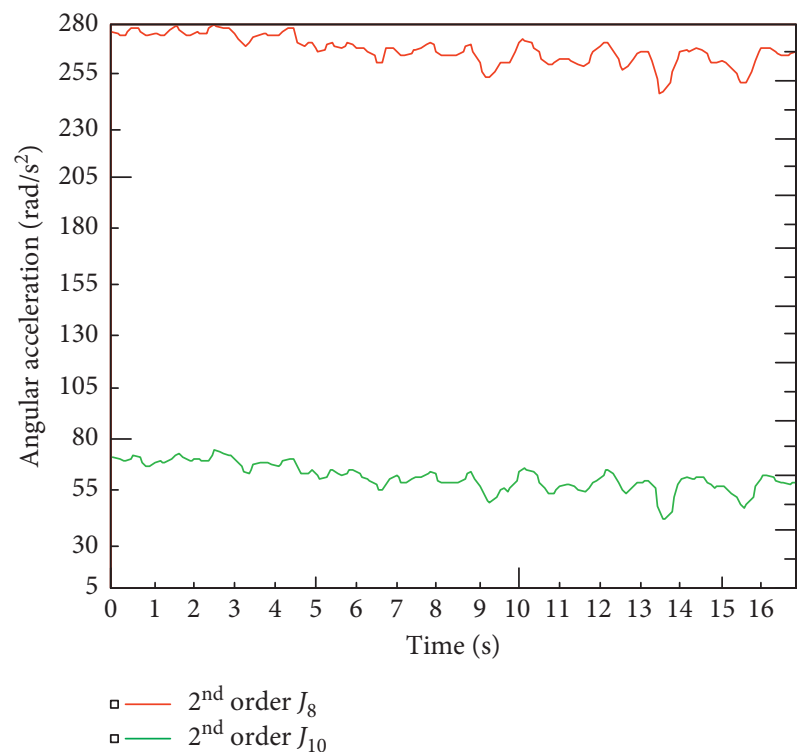

FIGURE 31: The $2^{\text {nd }}$ order angular acceleration curves of the primary flywheel and the input shaft of the gearbox matching the DMF with the bifilar CPVA under the idling condition.

TABle 7: Angular acceleration amplitudes under the idling condition of the powertrain matching the DMF with the bifilar CPVA.

\begin{tabular}{lcc}
\hline Items & $J_{8}\left(\mathrm{rad} / \mathrm{s}^{2}\right)$ & $J_{10}\left(\mathrm{rad} / \mathrm{s}^{2}\right)$ \\
\hline The overall angular acceleration & 510 & 150 \\
The $2^{\text {nd }}$ order angular acceleration & 270 & 65 \\
\hline
\end{tabular}

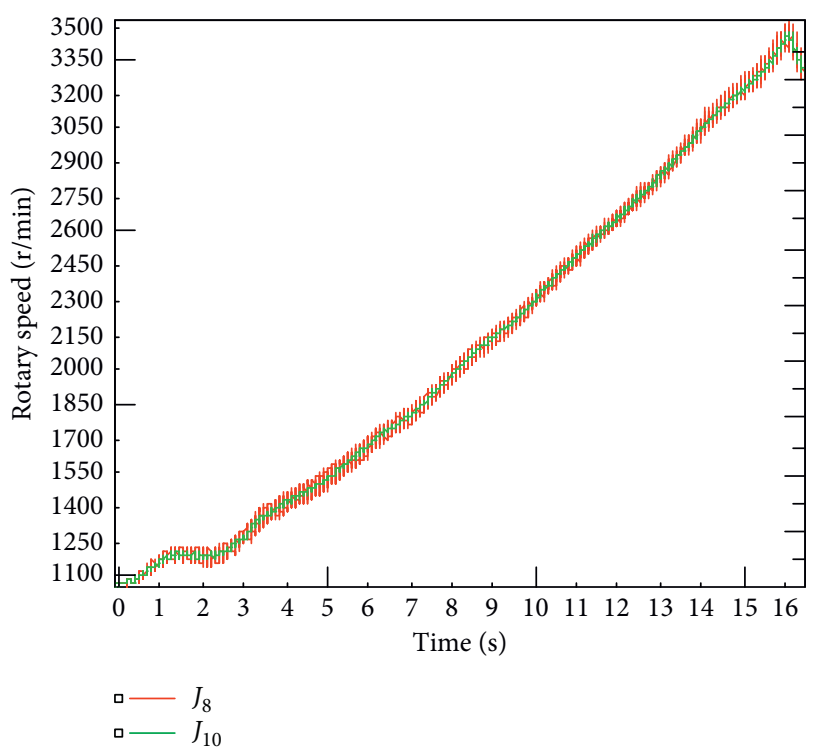

FIGURE 32: The rotary speed curves of the primary flywheel and the input shaft of the gearbox matching the DMF with the bifilar CPVA under driving conditions.

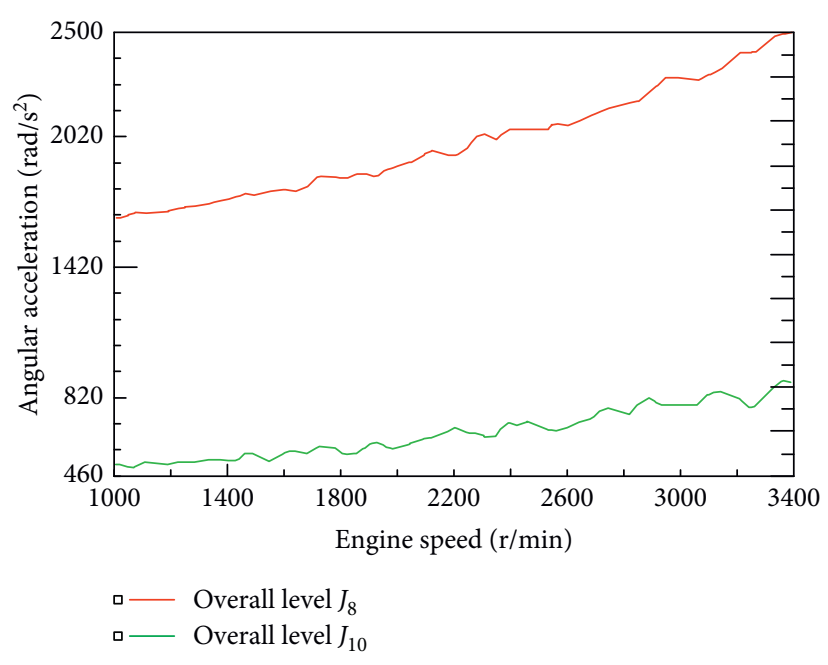

FIGURE 33: The overall angular acceleration curves of the primary flywheel and the input shaft of the gearbox matching the DMF with the bifilar CPVA under driving conditions.

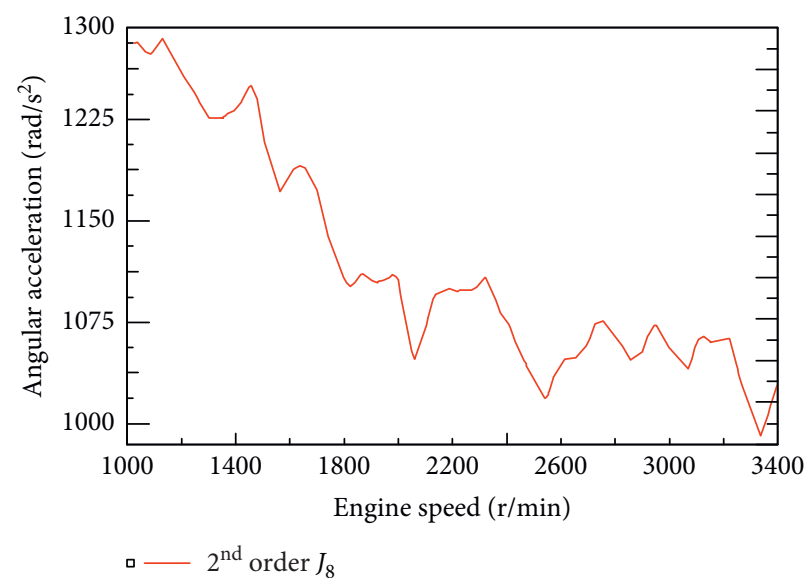

FIGURE 34: The $2^{\text {nd }}$ order angular acceleration curve of the primary flywheel matching with the bifilar CPVA under driving conditions.

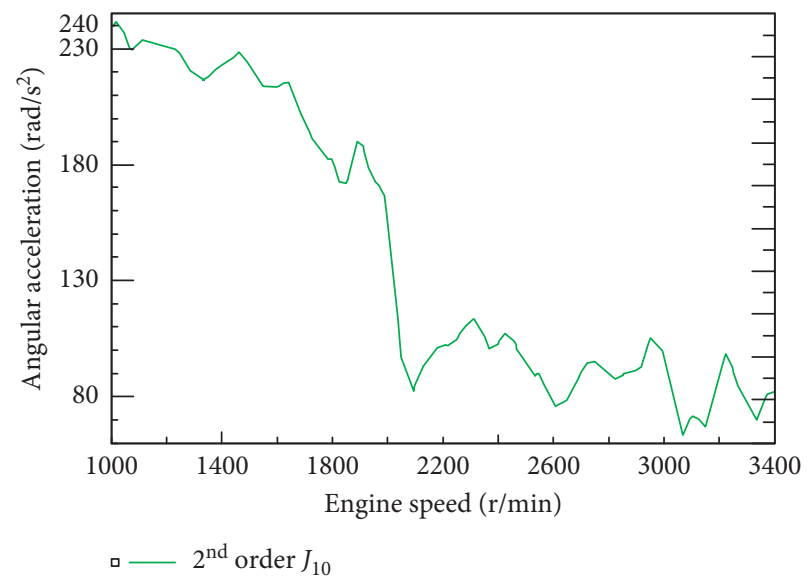

FIgURE 35: The $2^{\text {nd }}$ order angular acceleration curve of the input shaft of the gearbox matching the DMF with the bifilar CPVA under driving conditions. 
TABLE 8: The $2^{\text {nd }}$ order angular acceleration amplitudes under the driving condition for the powertrain matching the DMF with the bifilar CPVA.

\begin{tabular}{lcc}
\hline Engine speed $(\mathrm{r} / \mathrm{min})$ & $J_{8}\left(\mathrm{rad} / \mathrm{s}^{2}\right)$ & $J_{10}\left(\mathrm{rad} / \mathrm{s}^{2}\right)$ \\
\hline 1000 & 1300 & 240 \\
1500 & 1170 & 225 \\
2000 & 1055 & 152 \\
2500 & 1020 & 90 \\
3000 & 1060 & 93 \\
3400 & 1040 & 83 \\
\hline
\end{tabular}

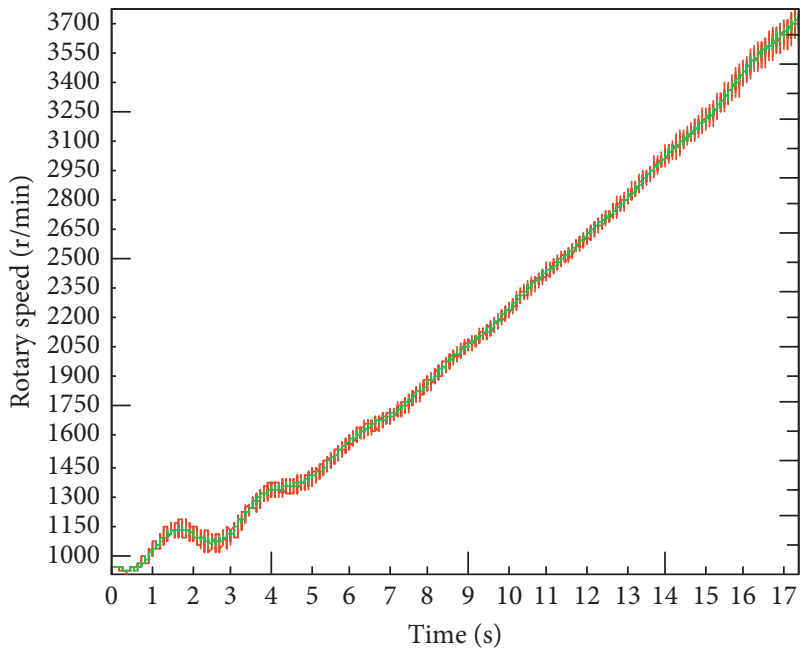

$$
\begin{aligned}
& \square-J_{8} \\
& \square-J_{10}
\end{aligned}
$$

FIGURE 36: The rotary speed curves of the primary flywheel and the input shaft of the gearbox matching the DMF under driving conditions.

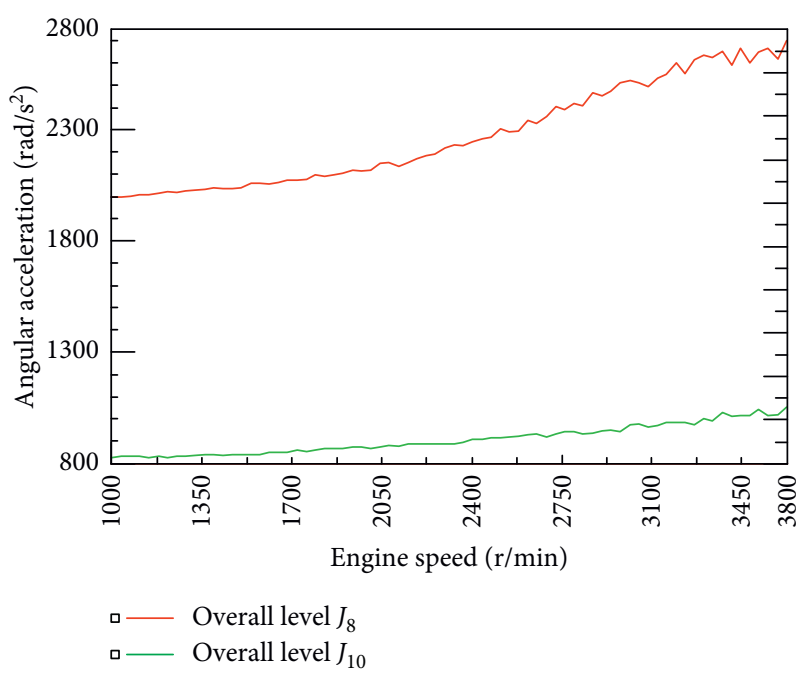

FIGURE 37: The overall angular acceleration curves of the primary flywheel and the input shaft of the gearbox matching the DMF under driving conditions.

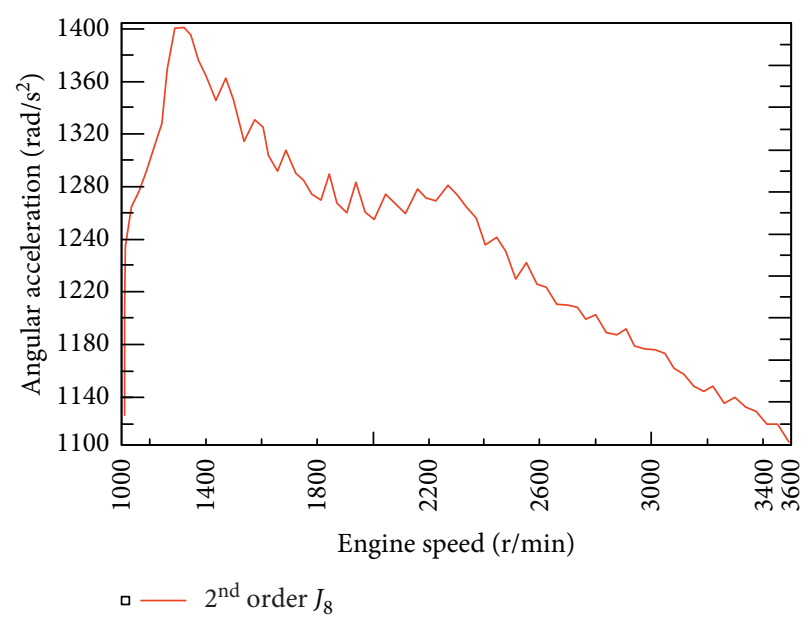

FIGURE 38: The $2^{\text {nd }}$ order angular acceleration curve of the primary flywheel matching without the CPVA under driving conditions.

excitation torques $T_{1}, T_{2}, T_{3}$, and $T_{4}$ are expressed as follows, where $T_{e}=5 \mathrm{~N} \cdot \mathrm{m}$ :

$$
\left\{\begin{array}{l}
T_{1}=T_{e} \sin \left(\left(\varepsilon \cdot \omega_{e} \cdot 2 \pi / 60\right) \cdot t\right) \\
T_{2}=T_{e} \sin \left(\left(\varepsilon \cdot \omega_{e} \cdot 2 \pi / 60\right) \cdot t+4 \pi\right) \\
T_{3}=T_{e} \sin \left(\left(\varepsilon \cdot \omega_{e} \cdot 2 \pi / 60\right) \cdot t+\pi\right) \\
T_{4}=T_{e} \sin \left(\left(\varepsilon \cdot \omega_{e} \cdot 2 \pi / 60\right) \cdot t+3 \pi\right) .
\end{array}\right.
$$

According to the parameters (Table 3), the simulation algorithm is carried out and the angular accelerations are obtained, as shown in Figure 6, where the blue curve represents the angular acceleration of the primary flywheel assembly $J_{8}$, the green curve represents the angular acceleration of the input shaft of the gearbox $J_{10}$ with the DMF, the red curve represents the angular acceleration of the input shaft of the gearbox $J_{10}$ with the DMF with the simple CPVA, and the black curve represents the angular acceleration of the input shaft of the gearbox $J_{10}$ with the DMF with the bifilar CPVA.

In the steady-state region, the overall angular acceleration amplitude of $J_{8}$ is $210 \mathrm{rad} / \mathrm{s}^{2}$, the overall angular acceleration amplitude of $J_{10}$ of DMF is $90 \mathrm{rad} / \mathrm{s}^{2}$, the overall angular acceleration amplitude of $J_{10}$ of DMF with simple CPVA is $53 \mathrm{rad} / \mathrm{s}^{2}$, and the overall angular acceleration amplitude of $J_{10}$ of DMF with bifilar CPVA is $30 \mathrm{rad} / \mathrm{s}^{2}$. In order to get a better view of the angular 


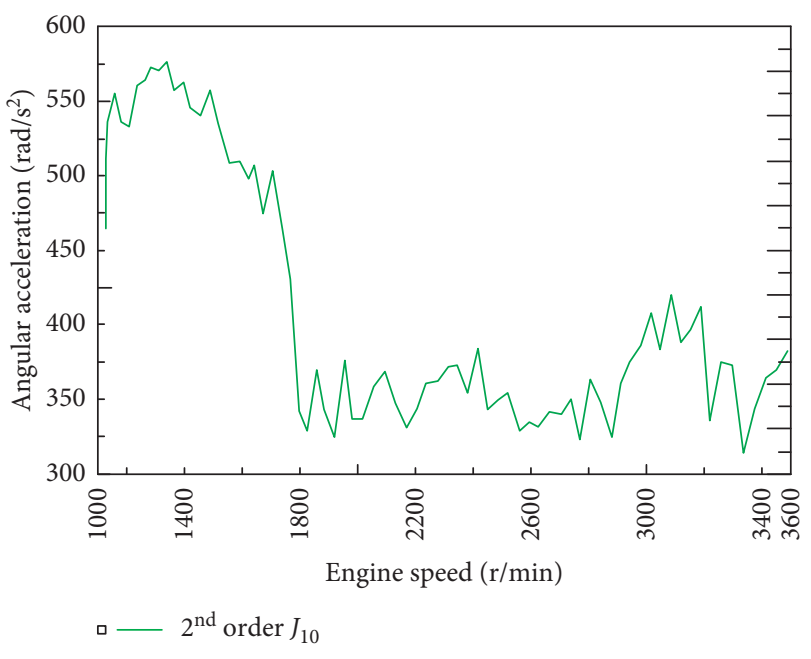

Figure 39: The $2^{\text {nd }}$ order angular acceleration curve of the input shaft of the gearbox matching the DMF under driving conditions.

TABLE 9: The $2^{\text {nd }}$ order angular acceleration amplitudes under the driving condition for the powertrain matching the DMF.

\begin{tabular}{lcc}
\hline Engine speed $(\mathrm{r} / \mathrm{min})$ & $J_{8}\left(\mathrm{rad} / \mathrm{s}^{2}\right)$ & $J_{10}\left(\mathrm{rad} / \mathrm{s}^{2}\right)$ \\
\hline 1000 & 1240 & 475 \\
1500 & 1380 & 550 \\
2000 & 1240 & 350 \\
2500 & 1230 & 350 \\
3000 & 1180 & 375 \\
3400 & 1130 & 360 \\
\hline
\end{tabular}

acceleration, the FFT of the acceleration signals has been plotted (see Figure 7).

4.2. Simulation under the Driving Condition. Under the driving condition, the simulations are carried out at five different speeds, that is, $\omega_{\mathrm{e}}=1000 \mathrm{r} / \mathrm{min}, \omega_{e}=1500 \mathrm{r} / \mathrm{min}$, $\omega_{\mathrm{e}}=2000 \mathrm{r} / \mathrm{min}, \omega_{e}=2500 \mathrm{r} / \mathrm{min}$, and $\omega_{e}=3000 \mathrm{r} / \mathrm{min}$. The excitation torques $T_{1}, T_{2}, T_{3}$, and $T_{4}$ are expressed as equation (47), where $T_{e}=25 \mathrm{Nm}$ and $\varepsilon=2$ The time-domain dynamic response results are shown in Figures 8-12, respectively, where the blue curve represents the angular acceleration of the primary flywheel assembly $J_{8}$, the green curve represents the angular acceleration of the input shaft of the gearbox $J_{10}$ with the DMF, the red curve represents the angular acceleration of the input shaft of the gearbox $J_{10}$ with the DMF with the simple CPVA, and the black curve represents the angular acceleration of the input shaft of the gearbox $J_{10}$ with the DMF with the bifilar CPVA. The FFT results of the acceleration signals are plotted in Figures 13-17 .

Under the working condition of engine speed $(1000 \mathrm{rpm})$, the overall angular acceleration amplitude of $J_{8}$ is $1350 \mathrm{rad} / \mathrm{s}^{2}$, the overall angular acceleration amplitude of $J_{10}$ of $\mathrm{DMF}$ is $800 \mathrm{rad} / \mathrm{s}^{2}$, the overall angular acceleration amplitude of $J_{10}$ of DMF with simple CPVA is $495 \mathrm{rad} / \mathrm{s}^{2}$, and the overall angular acceleration amplitude of $J_{10}$ of DMF with bifilar CPVA is $152 \mathrm{rad} / \mathrm{s}^{2}$. The FFT of the acceleration signals is obtained in Figure 13.

Under the working condition of engine speed $(1500 \mathrm{rpm})$, the overall angular acceleration amplitude of $J_{8}$ is $1065 \mathrm{rad} / \mathrm{s}^{2}$, the overall angular acceleration amplitude of $J_{10}$ of DMF is $250 \mathrm{rad} / \mathrm{s}^{2}$, the overall angular acceleration amplitude of $J_{10}$ of DMF with simple CPVA is $165 \mathrm{rad} / \mathrm{s}^{2}$, and the overall angular acceleration amplitude of $J_{10}$ of DMF with bifilar CPVA is $80 \mathrm{rad} / \mathrm{s}^{2}$. The FFT of the acceleration signals is been shown in Figure 14.

Under the working condition of engine speed $(2000 \mathrm{rpm})$, the overall angular acceleration amplitude of $J_{8}$ is $1030 \mathrm{rad} / \mathrm{s}^{2}$, the overall angular acceleration amplitude of $J_{10}$ of DMF is $115 \mathrm{rad} / \mathrm{s}^{2}$, the overall angular acceleration amplitude of $J_{10}$ of DMF with simple CPVA is $92 \mathrm{rad} / \mathrm{s}^{2}$, and the overall angular acceleration amplitude of $J_{10}$ of DMF with bifilar CPVA is $31 \mathrm{rad} / \mathrm{s}^{2}$. The FFT of the acceleration signals is given in Figure 15.

Under the working condition of engine speed $(2500 \mathrm{rpm})$, the overall angular acceleration amplitude of $J_{8}$ is $1030 \mathrm{rad} / \mathrm{s}^{2}$, the overall angular acceleration amplitude of $J_{10}$ of $\mathrm{DMF}$ is $95 \mathrm{rad} / \mathrm{s}^{2}$, the overall angular acceleration amplitude of $J_{10}$ of DMF with simple CPVA is $85 \mathrm{rad} / \mathrm{s}^{2}$, and the overall angular acceleration amplitude of $J_{10}$ of DMF with bifilar CPVA is $23 \mathrm{rad} / \mathrm{s}^{2}$. The FFT of the acceleration signals is plotted as Figure 16.

Under the working condition of engine speed (3000 rpm), the overall angular acceleration amplitude of $J_{8}$ is $1030 \mathrm{rad} / \mathrm{s}^{2}$, the overall angular acceleration amplitude of $J_{10}$ of DMF is $96 \mathrm{rad} / \mathrm{s}^{2}$, the overall angular acceleration amplitude of $J_{10}$ of DMF with simple CPVA is $85 \mathrm{rad} / \mathrm{s}^{2}$, and the overall angular acceleration amplitude of $J_{10}$ of DMF with bifilar CPVA is $21 \mathrm{rad} / \mathrm{s}^{2}$. The FFT of the acceleration signals is plotted as Figure 17.

4.3. Analysis of Simulation Results. Considering the simulation results of different speed conditions, the overall angular acceleration amplitudes of $J_{8}$ and $J_{10}$, which are the total dynamic responses of the power transmission system, are listed in Table 4 based on the simulation results. In the light of the FFT results, the $2^{\text {nd }}$ order angular acceleration amplitudes of $J_{8}$ and $J_{10}$ are summarized in Table 5 for engine speed $800 \mathrm{rpm}, 1000 \mathrm{rpm}, 1500 \mathrm{rpm}, 2000 \mathrm{rpm}, 2500 \mathrm{rpm}$, and $3000 \mathrm{rpm}$, and the $2^{\text {nd }}$ order harmonic frequencies are $26.7 \mathrm{~Hz}, 33.3 \mathrm{~Hz}, 50 \mathrm{~Hz}, 66.7 \mathrm{~Hz}, 83.3 \mathrm{~Hz}$, and $100 \mathrm{~Hz}$, respectively.

The simulation results both in the idling and driving conditions show that the DMF with the bifilar CPVA shows best effect on the attenuation of engine speed fluctuation in a speed range of 800 to $3000 \mathrm{r} / \mathrm{min}$. When the engine speed is lower than $1500 \mathrm{r} / \mathrm{min}$, the effect of the DMF with simple CPVA on the attenuation of engine speed fluctuation is better than that of the DMF; however, the damping effect is basically the same as the DMF when the engine speed is higher than $1500 \mathrm{r} / \mathrm{min}$. Furthermore, since the square root of the ratio of $R$ and $l$ from Table 2 is equal to 2 , the $2^{\text {nd }}$ harmonic-order excitation of the engine could be attenuated completely according to 
TABLE 10: Attenuation rate of the angular acceleration under the idling condition.

\begin{tabular}{lcc}
\hline Damper & $\begin{array}{c}\text { Attenuation rate of the overall angular acceleration } \\
(\%)\end{array}$ & $\begin{array}{c}\text { Attenuation rate of the } 2^{\text {nd }} \text { order angular acceleration } \\
(\%)\end{array}$ \\
\hline DMF & 53 & 50 \\
DMF with bifilar & 70.5 & 76 \\
CPVA & & 76 \\
\hline
\end{tabular}

TABLE 11: Attenuation rate of the $2^{\text {nd }}$ order angular acceleration under the driving condition.

\begin{tabular}{|c|c|c|}
\hline Condition & $\begin{array}{c}\text { DMF attenuation rate of the } 2^{\text {nd }} \text { order angular } \\
\text { acceleration }(\%)\end{array}$ & $\begin{array}{l}\text { DMF with the bifilar CPVA attenuation rate of the } 2^{\text {nd }} \text { order angular } \\
\text { acceleration (\%) }\end{array}$ \\
\hline $\begin{array}{l}1000 \\
\mathrm{r} / \mathrm{min}\end{array}$ & 62 & 82.5 \\
\hline $\begin{array}{l}1500 \\
\mathrm{r} / \mathrm{min}\end{array}$ & 60 & 80.8 \\
\hline $\begin{array}{l}2000 \\
\mathrm{r} / \mathrm{min}\end{array}$ & 71 & 85.6 \\
\hline $\begin{array}{l}2500 \\
\mathrm{r} / \mathrm{min}\end{array}$ & 71 & 91.1 \\
\hline $\begin{array}{l}3000 \\
\mathrm{r} / \mathrm{min}\end{array}$ & 68 & 91.2 \\
\hline $\begin{array}{l}3400 \\
\mathrm{r} / \mathrm{min}\end{array}$ & 68 & 92 \\
\hline
\end{tabular}

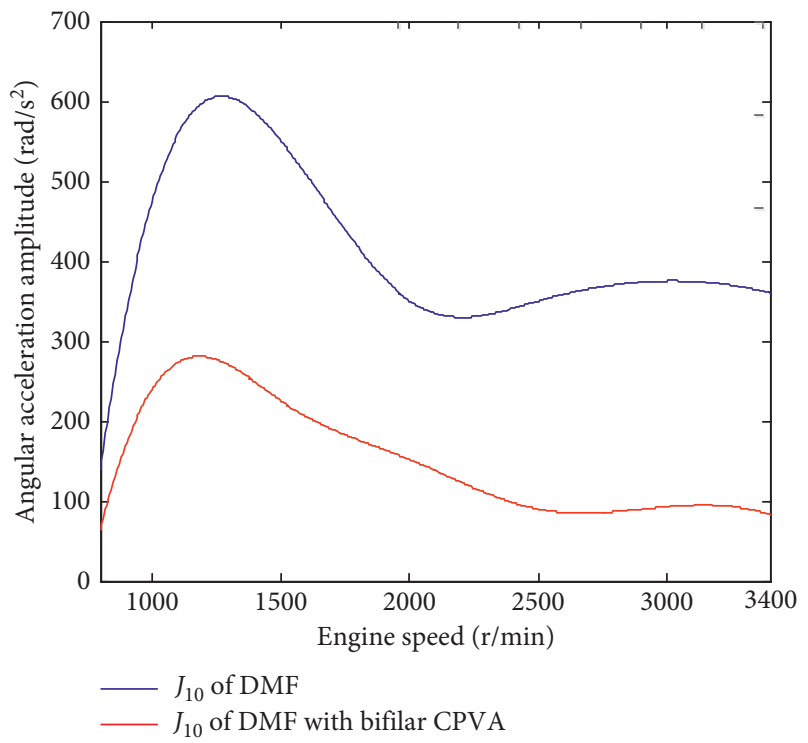

Figure 40: Comparison of the $2^{\text {nd }}$ order angular acceleration amplitudes of $J_{10}$ in the input shaft of the gearbox in the test.

equation (21). However, the simulation results demonstrate that the $2^{\text {nd }}$ harmonic-order excitation is not completely eliminated, which is attenuated by more than $90 \%$.

To summarize, the DMF with the bifilar CPVA shows the best damping effect in the whole speed range. Furthermore, in the low-speed region, the vibration reduction effect of the DMF with simple CPVA is better than that of the DMF, whereas they show the same damping performance in the high-speed region. In addition, the $\varepsilon^{\text {th }}$ harmonic-order excitation from the engine cannot be attenuated completely but can be attenuated by more than $90 \%$ when the square root of the ratio of $R$ and $l$ is equal to $\varepsilon$.

\section{Discussion on the Influence of $R$ and $l$ on Damping Performance}

The linear dynamic model of the bifilar CPVA suggests that the $\varepsilon^{\text {th }}$ harmonic-order excitation from the engine can theoretically be eliminated completely on the condition that the $R$ and $l$ of the bifilar CPVA satisfy equation (21). Al- 


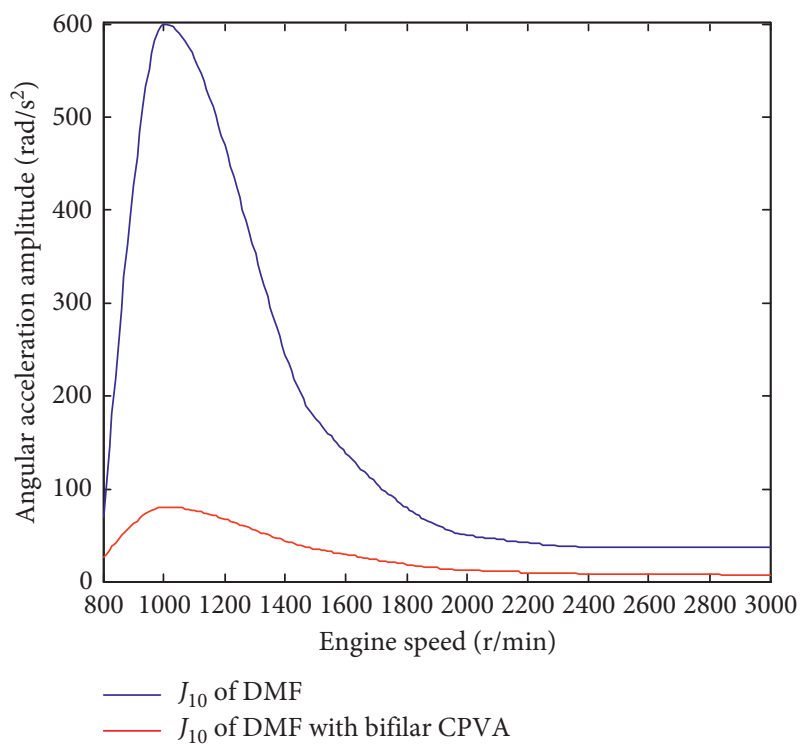

FIGURE 41: Comparison of the $2^{\text {nd }}$ order angular acceleration amplitudes of $J_{10}$ in the input shaft of the gearbox in the simulation.

though the simulation results demonstrate that the DMF with bifilar CPVA does not achieve the theoretical damping performance with $R$ and $l$ satisfying equation (21), and its damping effect on the torsional vibration from the engine is still excellent, which indicates that the ratio of $R$ to $l$ satisfying equation (21) has significant influence on the vibration reduction effect of the DMF with bifilar CPVA. Indeed, in the case, there are many combinations of $R$ and $l$.

Since the bifilar CPVA is installed on the driven plate of the DMF (Figure 18), the size of $R$ and $l$ of the bifilar CPVA is limited by the size of the driven plate. Let the inner and outer diameters of the driven plate be, respectively, $R_{1}$ and $R_{2}$, then

$$
0.5 R_{1} \leq R \leq 0.5 R_{2} .
$$

With reference to equation (21), the relationship between $R$ and $l$ satisfies

$$
\frac{R}{l}=\varepsilon^{2}
$$

Thus,

$$
\frac{1+\varepsilon^{2}}{2 \varepsilon^{2}} R_{1} \leq R+l \leq \frac{1+\varepsilon^{2}}{2 \varepsilon^{2}} R_{2} .
$$

The constraint conditions of the system are shown in equation (50), the design variable of the system is $R+l$, and then, the objective function of the system is given by

$$
f\left(\ddot{\alpha}_{10}\right)=\min f\left(\dot{\alpha}_{1} \ldots \dot{\alpha}_{11}, \alpha_{1} \ldots \alpha_{11}, \dot{\phi}, \phi\right),
$$

where $f\left(\dot{\alpha}_{1} \ldots \dot{\alpha}_{11}, \alpha_{1} \ldots \alpha_{11}, \dot{\phi}, \phi\right)$ is the system state equation from equations (45) and (46): $R_{1}=132 \mathrm{~mm}$ and $R_{2}=154 \mathrm{~mm}$.

For the four-cylinder and four-stroke engine, $\varepsilon$ is equal to 2 . Based on the above model, the angular acceleration amplitude of $J_{10}$ varying with the structural parameter $R+l$ of the bifilar CPVA can be plotted in Figure 19 by using Newton's method.
The result shows that, under the above constraints, the amplitude of the angular acceleration of $J_{10}$ is inversely proportional to $R+l$; that is, the damping effect of the DMF with the bifilar CPVA is directly proportional to $R+l$.

\section{Real Vehicle Tests}

In this section, the real vehicle tests are carried out for power transmissions matching the DMF with the bifilar CPVA and the DMF. The test vehicle is a Fengguang series SUV of Dongfeng Xiaokang automobile company. As for the test vehicle, the maximum torque and the maximum speed of the four-cylinder and four-stroke engine are $220 \mathrm{Nm}$ and $6000 \mathrm{r} / \mathrm{min}$, respectively, which is equipped with a CVT from Aisin Seiki Company. Figure 20 shows the sensor layout on the power transmission. Two electromagnetic rotating speed sensors, in which the model number is ONOSOKKI-MP-910, are mounted on the housing of the gearbox, where the no. 1 sensor is pointed to the signal gear on the primary flywheel and the no. 2 sensor is pointed to the signal gear on the input shaft of the gearbox, and the arrangement details of these two sensors are shown in Figure 21. It should be noted that there was no signal gear on the input shaft of the gearbox. In order to test the rotational speed of the input shaft, a signal gear was processed and installed on the input shaft of the gearbox. The signals of rotating speed are acquired by Siemens data acquisition instrument (Figure 22), of which the type is LMS SCADAS302VB.

During the tests, the rotating speed signals of the output shaft of the engine and the input shaft of the gearbox are tested under the idling and driving conditions, and the angular acceleration signals can be obtained by derivative of speed signals to time. The rotating speed signals of the electromagnetic rotating speed sensor are similar to the sinusoidal wave. Let the rotating speed of the gear be $\omega(\mathrm{r} / \mathrm{min})$, the number of teeth of the gear be $z_{g}$, 
and the frequency of the signals be $f(\mathrm{~Hz})$, then $\omega$ will be $\omega=60 * f / z_{g}$. Meanwhile, the collected data were processed by Siemens LMS Test.Lab 14A, and then, tracking settings for two signal channels are shown in Figure 23, where the number of teeth of the signal gear on the primary flywheel is 133 and the number of teeth of the signal gear on the input shaft of the gearbox is 60 . The acquisition parameters are shown in Figure 24, where the acquisition bandwidth is $800 \mathrm{~Hz}$ and sampling frequency is $2000 \mathrm{~Hz}$. In Figure 25, tachol and tacho2 are the rotating speed signal channel of the primary flywheel and the rotating speed signal channel of the input shaft of the transmission, respectively.

The angular acceleration of the primary flywheel and the angular acceleration of the input shaft of the gearbox are measured under the idling condition and driving condition during the real vehicle experiment. Under the idling condition, the engine speed is maintained around $750 \mathrm{r} / \mathrm{min}$ for about 20 seconds; simultaneously, the angular acceleration of the primary flywheel and the angular acceleration of the input shaft of the gearbox are measured by the two electromagnetic rotating speed sensors. Under the driving condition, the gearbox is in the forward gear position, and then, the engine speed is evenly accelerated from $1000 \mathrm{r} / \mathrm{min}$ to about $3500 \mathrm{r} / \mathrm{min}$ by stepping on the accelerator pedal and the whole process is about 17 seconds. During the change in engine speed, the angular acceleration of the primary flywheel and the angular acceleration of the input shaft of the gearbox are recorded by the two electromagnetic rotating speed sensors. Under the driving condition, the engine speed range in this test is mainly based on the following two factors:

(1) The maximum speed of the engine is $6000 \mathrm{r} / \mathrm{min}$, and then the engine speed range, $1000 \mathrm{r} / \mathrm{min} \sim 3400 \mathrm{r} /$ $\mathrm{min}$, is the common engine speed range, which basically covers the low-speed zone and high-speed zone.

(2) At present, there is no mass production capacity of DMF with bifilar CPVA in China. The DMF with bifilar CPVA used in this experiment is a sample, and the reliability and fatigue experiments have not been done, so this experiment does not cover the whole engine speed range.

In the following test data analysis, all acceleration amplitudes refer to the half of the peak-to-peak amplitudes.

6.1. Real Vehicle Test under the Idling Condition. For the powertrain with the DMF, the engine speed is around $750 \mathrm{r}$ / min under the idling condition, as shown in Figure 26, in which the red and green curves represent the engine speed and the speed of the input shaft of the gearbox, respectively. Obviously, the fluctuating range of the engine speed is from $730 \mathrm{r} / \mathrm{min}$ to $790 \mathrm{r} / \mathrm{min}$. Figure 27 shows the overall angular acceleration under the idling condition, in which the red and green curves, respectively, present the overall angular acceleration of the primary flywheel and the input shaft of the gearbox. Furthermore, the $2^{\text {nd }}$ order angular acceleration can be obtained by harmonic tracking, as shown in Figure 28, where the red and green curves, respectively, present the $2^{\text {nd }}$ order angular acceleration of the primary flywheel and the input shaft of the gearbox.

According to the test results for the powertrain with the DMF under the idling condition, the specific data are shown in Table 6, which indicates that the overall and $2^{\text {nd }}$ angular acceleration of the engine are attenuated by $53 \%$ and $50 \%$, respectively.

For the powertrain matching the DMF with the bifilar $\mathrm{CPVA}$, the engine speed is also around $750 \mathrm{r} / \mathrm{min}$ under the idling condition, as shown in Figure 29, in which the red and green curves represent the engine speed and the input shaft of the gearbox rotary speed, respectively; obviously, the fluctuating range of the engine speed is from $720 \mathrm{r} / \mathrm{min}$ to $770 \mathrm{r} / \mathrm{min}$. Figure 30 shows the overall angular acceleration under the idling condition, in which the red and green curves, respectively, present the overall angular acceleration of the primary flywheel and the input shaft of the gearbox. Similarly, the $2^{\text {nd }}$ order angular acceleration by harmonic tracking is shown in Figure 31, where the red and green curves, respectively, present the $2^{\text {nd }}$ order angular acceleration of the primary flywheel and the input shaft of the gearbox.

According to the test results, for the powertrain matching the DMF with the bifilar CPVA under the idling condition, the specific data are listed in Table 7, which suggests that the overall and $2^{\text {nd }}$ angular acceleration of the engine are attenuated by $70.5 \%$ and $76 \%$, respectively.

6.2. Real Vehicle Test under the Driving Condition. For the powertrain matching the DMF with the bifilar CPVA, under the driving condition, the range of the engine speed is $1000 \mathrm{r} / \mathrm{min}-3500 \mathrm{r} / \mathrm{min}$, as shown in Figure 32, in which the red and green curves represent the engine speed and the rotary speed of the input shaft of the gearbox, respectively. Figure 33 shows the overall angular acceleration under the driving condition, and Figures 34 and 35 depict the $2^{\text {nd }}$ order angular acceleration of the primary flywheel and the input shaft of the gearbox.

During the test, the engine speed increased from $1000 \mathrm{r} /$ $\mathrm{min}$ to $3500 \mathrm{r} / \mathrm{min}$, the overall angular acceleration is affected by the speed of the accelerator pedal, and thus, the $2^{\text {nd }}$ order angular acceleration can more accurately reflect the damping effect. The specific data of the $2^{\text {nd }}$ order angular acceleration are listed in Table 8 . When the engine speed is $1000 \mathrm{r} / \mathrm{min}, 1500 \mathrm{r} / \mathrm{min}, 2000 \mathrm{r} / \mathrm{min}, 2500 \mathrm{r} / \mathrm{min}, 3000 \mathrm{r} / \mathrm{min}$, and $3400 \mathrm{r} / \mathrm{min}$, the $2^{\text {nd }}$ angular acceleration of the engine is attenuated by $82.5 \%, 80.8 \%, 85.6 \%, 91.1 \%, 91.2 \%$, and $92 \%$, respectively.

For the powertrain with the DMF, under the driving condition, the range of engine speed is $1000 \mathrm{r} / \mathrm{min}-3700 \mathrm{r} /$ min. As shown in Figure 36, the red and green curves represent the engine speed and the rotary speed of the input shaft of the gearbox, respectively. Figure 37 shows the overall angular acceleration under the driving condition. Moreover, the $2^{\text {nd }}$ order angular acceleration by harmonic tracking is shown in Figures 38 and 39 . 
The specific data of the $2^{\text {nd }}$ order angular acceleration are shown in Table 9. When the engine speed is $1000 \mathrm{r} / \mathrm{min}, 1500 \mathrm{r} /$ $\mathrm{min}, 2000 \mathrm{r} / \mathrm{min}, 2500 \mathrm{r} / \mathrm{min}, 3000 \mathrm{r} / \mathrm{min}$, and $3400 \mathrm{r} / \mathrm{min}$, respectively, the $2^{\text {nd }}$ angular acceleration of the engine is attenuated by $62 \%, 60 \%, 71 \%, 71 \%, 68 \%$, and $68 \%$, respectively.

6.3. Discussion on Real Vehicle Test Result. The experimental data under idling and driving conditions are summarized in Tables 10 and 11, respectively, and the comparison of the $2^{\text {nd }}$ order angular acceleration amplitudes of $J_{10}$ in the input shaft of the gearbox in the test is plotted in Figure 40, which represent that the DMF with bifilar CPVA shows a better damping performance than DMF under idling and driving conditions. Moreover, regarding the DMF, the $2^{\text {nd }}$ angular acceleration amplitude of the input shaft of the gearbox is rapidly reduced with the engine speed from $750 \mathrm{r} / \mathrm{min}$ to $2000 \mathrm{r} / \mathrm{min}$ but basically stable with the engine speed from $2000 \mathrm{r} / \mathrm{min}$ to $3400 \mathrm{r} / \mathrm{min}$. On the other hand, considering the DMF with the bifilar CPVA, the $2^{\text {nd }}$ order angular acceleration amplitude of the input shaft of the gearbox is uniformly attenuated with the engine speed from $750 \mathrm{r} / \mathrm{min}$ to $3400 \mathrm{r} / \mathrm{min}$.

Referring to the simulation data in Table 5, the comparison of the $2^{\text {nd }}$ order angular acceleration amplitudes of $J_{10}$ in the input shaft of the gearbox in the simulation is plotted in Figure 41. The simulated excitation torque value is not the actual excitation value, the actual excitation frequencies are more complex, and the angular acceleration amplitudes cannot be used as a reference in the comparison of experimental results and simulation results; however, the two comparisons of the $2^{\text {nd }}$ order angular acceleration amplitudes of $J_{10}$ (Figures 40 and 41) demonstrate basically the same trend; that is, the $2^{\text {nd }}$ angular acceleration amplitudes of the input shaft of the gearbox are rapidly attenuated as for the DMF and the DMF with bifilar CPVA with the engine speed lower than $2000 \mathrm{r} / \mathrm{min}$. Nevertheless, when the engine speed is higher than $2000 \mathrm{r} / \mathrm{min}$, the $2^{\text {nd }}$ order angular accelerations of the input shaft of the gearbox with the DMF are basically stable; on the contrary, the $2^{\text {nd }}$ order angular accelerations of the input shaft of the gearbox matching the DMF with bifilar CPVA are still attenuated. In addition, the DMF with bifilar CPVA shows a better damping performance than DMF in the whole test speed range. The regular pattern shows that the experimental results are basically consistent with the simulation results, which validate the validity of the proposed dynamic model of the DMF with the bifilar CPVA.

There are few published studies about DMF and CPVA as an ensemble, and in [12], only numerical simulation was done and no experimental verification was carried out. In addition, the real vehicle experiment of a clutch with CPVA was executed in [13]; however, the testing condition of this experiment was engine speed at $850 \mathrm{r} / \mathrm{min}$ and the test results showed that the $2^{\text {nd }}$ order rotational speed amplitude was attenuated by $90 \%$ under the condition. Compared with the rotational speed, the angular acceleration can better characterize the torsional vibration of the powertrain. In this paper, the engine speed range of real vehicle experiment is wider, and the damping performance of DMF and DMF with bifilar CPVA is compared and analyzed by angular acceleration from the test results, which makes up for the lack of verification work of real vehicle experiment in previous research.

\section{Conclusions}

This study addresses the linear and the nonlinear dynamic model of the DMF with the bifilar CPVA. The linear dynamic model of the DMF with the bifilar CPVA reveals the vibration reduction principle and the importance of the structural parameters of $R$ and $l$. Furthermore, the dynamic model of the powertrain based on the nonlinear dynamic model of the DMF with the bifilar CPVA is developed, and the dynamic responses are simulated through the speed range of $800-3000 \mathrm{r} / \mathrm{min}$. Moreover, the influence of $R$ and $l$ on the damping performance is discussed on the basis of the dynamic model, and subsequently, the validity of the model is verified by the real vehicle tests under idling and driving conditions. The main conclusions of this research are summed up as follows:

(1) The bifilar CPVA can be regarded as a dynamic unit in which the natural frequency varies with the rotational speed. The linear dynamic model shows that the $\varepsilon^{\text {th }}$ harmonic-order torsional vibration can be eliminated completely when the square root of the ratio of $R$ and $l$ is equal to $\varepsilon$; however, the simulation and test results indicate that the $\varepsilon^{\text {th }}$ harmonic-order torsional vibration can only be attenuated by $80 \%$ to $90 \%$ and not be isolated from the transmission completely.

(2) Under the constraints of the installation size and the ratio of $R$ to $l$, the angular acceleration amplitude of the input shaft of the gearbox is inversely proportional to $R+l$; that is, the damping effect of the DMF with the bifilar CPVA is directly proportional to $R+l$.

(3) In the whole engine speed region, the DMF with bifilar CPVA possesses the best damping performance among the three kinds of torsional dampers, which are the DMF, the DMF with simple CPVA, and the DMF with bifilar CPVA. If the square root of the ratio of $R$ and $l$ is equal to $\varepsilon$, for the DMF, the $\varepsilon^{\text {th }}$ order angular acceleration amplitude of the input shaft of the gearbox can be rapidly attenuated by the DMF with the engine speed lower than $2000 \mathrm{r} / \mathrm{min}$, but it is basically stable with the engine speed higher than $2000 \mathrm{r} / \mathrm{min}$. For the DMF with the bifilar CPVA, the $\varepsilon^{\text {th }}$ order angular acceleration amplitude of the input shaft of the gearbox can be continuously attenuated in the whole engine speed region.

(4) The simulation and test results suggest that the angular acceleration amplitudes of the primary flywheel are hardly affected by the DMF and the DMF with the bifilar CPVA.

(5) The nonlinear dynamic model of the DMF with the bifilar CPVA contains the dynamic parameters of the 
DMF and the structural parameters of the bifilar CPVA. In this paper, the influence of $R$ and $l$ on the damping performance of the system is only discussed theoretically, and the comparison tests of different $R$ and $l$ have not been carried out due to the limited experimental conditions and the difficulty in making samples.

(6) The model and methods discussed here can offer guidelines for the design and optimization of DMF with bifilar CPVA and similar shock absorbers for rotating machinery systems.

(7) The simulation and test results show that bifilar CPVA can further improve the damping performance of DMF by attenuating the $2^{\text {nd }}$ order rotational speed fluctuation. By analyzing the influence of the performance parameters of bifilar CPVA on the design model of DMF, the method of improving the performance parameters of DMF will be found to attenuate the rotational speed fluctuation from the engine in other orders and the damping performance of DMF with bifilar CPVA can be further enhanced, which will be the focus of the future research.

\section{Data Availability}

The data used to support the findings of this study are included within the article.

\section{Conflicts of Interest}

The authors declare that there are no conflicts of interest with respect to the research, authorship, and publication of this article.

\section{Authors' Contributions}

Lei Chen conceptualized the study, investigated the study, and wrote the original draft. Lei Chen and Jianming Yuan prepared the methodology. Jinmin $\mathrm{Hu}$ analyzed using the software. Hang Cai and Jianming Yuan validated the study. Jianming Yuan reviewed and edited the manuscript. Lei Chen and Jinmin $\mathrm{Hu}$ obtained funding acquisition. All authors have read and agreed to the published version of the manuscript.

\section{Acknowledgments}

This research was funded by the National Natural Science Foundation of China (Grant no. 51405355) and the Provincial Science and Technology Program of Guangdong Province (Grant no. 2018B030323013).

\section{References}

[1] G. Wu and W. Luan, "Review of dynamic research for NVH problems related to automotive driveline," Journal of $\mathrm{Me}$ chanical Engineering, vol. 49, no. 24, pp. 108-119, 2013.

[2] N. Pavel, P. Ales, and Z. Martin, "Investigating the influence of computational model complexity on noise and vibration modeling of powertrain," Journal of Vibroengineering, vol. 18, no. 1, pp. 378-393, 2016.
[3] Y. D. Hao, Z. C. He, G. Y. Li, E. Li, and Y. Y. Huang, "Uncertainty analysis and optimization of automotive driveline torsional vibration with a driveline and rear axle coupled model," Engineering Optimization, vol. 50, no. 11, pp. 1871-1893, 2018.

[4] F. Jian, K. Diao, and X. Wang, "Experimental study and simulation analysis on torsional vibration characteristic of 3cylinder engine driveline system," Journal of Vibration, Measurement \& Diagnosis, vol. 40, no. 1, pp. 115-121, 2020.

[5] H. Ahmed, M. Eliot, and T. Stephanos, "A Study on torsional vibration attenuation in automotive drivetrains using absorbers with smooth and non-smooth nonlinearities," Journal of Applied Mathematical Modeling, vol. 46, pp. 674-690, 2017.

[6] W. B. Shang guan, X. L. Liu, Y. Yin, and S. Rakheja, "Modeling of automotive driveline system for reducing gear rattles," Journal of Sound and Vibration, vol. 416, pp. 136-153, 2018.

[7] L. Chen, W. Shi, Z. Chen, G. Liu, and H. Liu, "An analysis on torsional characteristics of Dual Mass Flywheel and vehicle test study," Automotive Engineering, vol. 41, no. 11, pp. 1294-1300, 2019.

[8] L. Song, Z. Zhi, L. Zeng, and H. Tian, "Design research on the safety device of automobile Dual Mass Flywheel based on auto-lock theory," Journal of Mechanical Engineering, vol. 51, no. 4, pp. 141-147, 2015.

[9] Y. Wang, X. Qin, S. Huang, and S. Deng, "Design and analysis of a multi-stage torsional stiffness dual mass flywheel based on vibration control," Applied Acoustics, vol. 104, pp. 172-181, 2016.

[10] M. Cirelli, J. Gregori, P. P. Valentini, and E. Pennestrí, “A Design chart approach for the tuning of parallel and trapezoidal bifilar centrifugal pendulum," Mechanism and Machine Theory, vol. 140, pp. 711-729, 2019.

[11] W. Li, L. Yan, and W. Shi, "Analysis of isolation of the torsional vibration of DMF -CS with centrifugal pendulumtype absorber," Journal of China Mechanical Engineering, vol. 20 , no. 15, pp. 1787-1790, 2009.

[12] H. Wu and G. Wu, "Centrifugal pendulum vibration absorber and its application to torsional vibration damper with large angular displacement," Automotive Engineering, vol. 39, no. 12, pp. 1409-1416, 2017.

[13] M. Hässler, A. Kooy, R. Welter, and V. Lichtenwald, "Clutch disc with centrifugal pendulum absorber," Auto Tech Review, vol. 5, no. 4, pp. 26-31, 2016.

[14] S.-C. S. Seong and G. W. Kim, “Torsional vibration isolation performance evaluation of centrifugal pendulum absorbers for clutch dampers," Transactions of the Korean Society for Noise and Vibration Engineering, vol. 26, no. 4, pp. 436-442, 2016.

[15] L. Chen, W. Shi, and Z. Chen, "Modeling of idle speed transmission based on unit modeling method and performance comparison of various torsional dampers," Journal of Central South University (Science and Technology), vol. 51, no. 3, pp. 842-852, 2020.

[16] M. Rao and C. Sujatha, "Design of centrifugal pendulum vibration absorber to reduce the axial vibration of rotating shafts for multiple orders," SAE International Journal of Passenger Cars, vol. 13, no. 2, pp. 1-23, 2020.

[17] A. S. Alsuwaiyan and S. W. Shaw, "Performance and dynamic stability of general-path centrifugal pendulum vibration absorbers," Journal of Sound and Vibration, vol. 252, no. 5, pp. 791-815, 2002.

[18] C. Shi and R. G. Parker, "Modal properties and stability of centrifugal pendulum vibration absorber systems with equally 
spaced, identical absorbers," Journal of Sound and Vibration, vol. 331, no. 21, pp. 4807-4824, 2012.

[19] M. C. Cirelli, E. Valentini, and E. Pennestr, "The tuning conditions for circular, cycloidal and epicycloidal centrifugal pendula: a unified cartesian approach," Mechanism and Machine Theory, vol. 150, pp. 1-26, 2020.

[20] J. Mayet and H. Ulbrich, "Tautochronic centrifugal pendulum vibration absorbers," Journal of Sound and Vibration, vol. 333, no. 3, pp. 711-729, 2014.

[21] C. Shi and R. G. Parker, "Optimal tuning of centrifugal pendulum vibration absorbers," in Proceedings of the ASME 2013 International Design Engineering Technical Conferences and Computers and Information in Engineering Conference, vol. 8, Portland, OR, USA, August 2013.

[22] V. Pier Paolo, M. Cirelli, and Simone Di Donato, "The compliant centrifugal pendulum as the vibration absorber with second-order elasto-kinematic approximation," Journal of Vibration and Control, vol. 20, 2020.

[23] L. Chen, R. Zeng, and Z. Jiang, "Nonlinear dynamical model of an automotive dual mass flywheel," Advances in Mechanical Engineering, vol. 7, no. 6, pp. 1-11, 2015.

[24] J. E. Mottershead and R. Stanway, "Identification of NthPower velocity damping," Journal of Sound and Vibration, vol. 105, no. 2, pp. 309-319, 1986. 